\title{
VARIATIONAL SUBMANIFOLDS OF EUCLIDEAN SPACES
}

\author{
D. KRUPKA, Z. URBAN, AND J. VOLNÁ
}

\begin{abstract}
Systems of ordinary differential equations (or dynamical forms in Lagrangian mechanics), induced by embeddings of smooth fibered manifolds over one-dimensional basis, are considered in the class of variational equations. For a given non-variational system, conditions assuring variationality (the Helmholtz conditions) of the induced system with respect to a submanifold of a Euclidean space are studied, and the problem of existence of these "variational submanifolds" is formulated in general and solved for second-order systems. The variational sequence theory on sheaves of differential forms is employed as a main tool for analysis of local and global aspects (variationality and variational triviality). The theory is illustrated by examples of holonomic constraints (submanifolds of a configuration Euclidean space) which are variational submanifolds in geometry and mechanics.
\end{abstract}

KeYwords: Euler-Lagrange equations; Helmholtz conditions; variational sequence; sheaf cohomology; fibered manifold; jet; submanifold

MSC: 49Q99 58A15 58A20 14F40 70F20

\section{INTRODUCTION}

Our main objective in this article is to study restrictions of simple integral variational functionals, defined on Euclidean spaces, to submanifolds of these spaces. The submanifolds may have specific topological structure; the question is what can be said in general about the influence of the topology on variationality of differential equations, given on submanifolds, and on the existence and structure of the corresponding local and global variational principles. Specifically, given a system of (ordinary) differential equations on a Euclidean space, not necessarily variational, we search for variational submanifolds on which the constrained system becomes (locally or globally) variational. This concept within the theory of variational differential equations is new, and belongs to transformations of differential equations into variational ones; see Voicu and Krupka 14, and references therein.

The method how to understand of what is going on when we restrict an integral variational functional to a submanifold is the variational sequence theory. Recall for example that a basic concept of the calculus of variations, the Euler-Lagrange mapping, can be constructed (for variational functionals on fibered spaces) as the quotient mapping of the exterior derivative operator $d$, acting on differential forms, by the restriction of $d$ to the so called contact forms. This construction can, in principle, be described in a simple way: the de Rham sequence on the corresponding underlying manifold should be factored through its contact subsequence. Then it turns out, in particular, that one of the quotient morphisms coincides with the Euler-Lagrange mapping. The quotient (sheaf) sequence, the variational sequence, then can be used to study the local and global properties of the Euler-Lagrange 
mapping. Note, however, that the variational sequence includes much more relevant local and global information on the variational functionals on fibered spaces.

To study the variationality concepts on submanifolds, we shall focus our attention to the restriction of variational sequences, defined over fibrations $\mathbb{R} \times \mathbb{R}^{m}$ over the real line $\mathbb{R}$, representing time, to subfibrations $\mathbb{R} \times Q$, where $Q$ is an embedded submanifold of $\mathbb{R}^{m}$ (the constraint submanifold). This structure of underlying spaces corresponds from variational point of view to simple integral problems for curves from $\mathbb{R}$ to $\mathbb{R}^{m}$ or from $\mathbb{R}$ to $Q$.

Section 2 is devoted to basic facts, related with the notion of a contact differential form on some jet prolongation of a fibered manifold. We extend these concepts to fibered manifolds smoothly embedded in a Euclidean space, and describe properties of the pull-back of contact forms.

Section 3 is divided in three parts. First we give a brief summary of basic assertions of the theory of variational sequences over one-dimensional bases (real line $\mathbb{R}$, circle $S^{1}$ ). Next we study a modification of the variational sequence theory to embedded submanifolds in Euclidean spaces. Our main purpose is to characterize global aspects of extremal equations subjected to holonomic constraints. In the third part we recall relations of variational sequences and the calculus of variations.

In Section 4 we investigate systems of second-order ordinary differential equations, which become (locally or globally) variational when contracted to an embedded submanifold of the Euclidean space $\mathbb{R}^{m}$. The general problem to be solved is twofold: (a) to determine the structure of a system such that the induced system is variational, and (b) to characterize the class of submanifolds, which induce variational systems. Our main results are summarized in two theorems, stating the corresponding Helmholtz-type conditions.

Section 5 contains some examples of holonomic systems and the corresponding variational submanifolds. On one side we discuss the structure of constrained systems and obstructions for global variationality for topologically non-equivalent, embedded submanifolds of $\mathbb{R}^{3}$ (the sphere $S^{2}$ and the Möbius strip $M_{r, a}$ ). On the other side, we study the existence of variational submanifolds for concrete examples of second-order mechanical systems (e.g. the dumped oscillator, the gyroscopictype system) and, in particular, systems possessing the spheres $S^{1}$ and $S^{2}$ as the variational submanifolds are obtained.

Our approach to variational submanifolds in its simplest setting describes basic ideas of the theory of variational submanifolds as a part of the variational sequence theory. It can be extended in several directions, for instance to different underlying structures, related with multiple integrals, partial differential equations, variational principles in field theory with constraints, and variational sequences on Grassmann fibrations, where the variationality conditions of the Helmholtz type are available.

Basic reference for variational sequence theory in fibered manifolds over onedimensional basis is Krupka [6] (for Grassmann fibrations see Urban and Krupka 12 and Urban [13). For the Helmholtz variationality conditions in mechanics and field theory we refer to Anderson and Duchamp [1], Krupka [4], and Sarlet [10]. General variational sequence theory is explained in Krupka [5], [7. In all these sources also generalities on global variational principles in fibered spaces can be found; for basic notions and assertions see also Brajerčík and Krupka [2, Krupka and Saunders [8], Takens [11, and Volná and Urban [15]. 


\section{Contact FORMS ON EMBEDDED SUBMANIFOLdS}

Suppose that $\pi: Y \rightarrow X$ is a fibered manifold of dimension $m+1, m \geq 0$, over a one-dimensional base manifold $X$ (fibered mechanics). For any integer $r \geq 0$, $J^{r} Y$ denotes the $r$-jet prolongation of $Y$, and $\pi^{r}: J^{r} Y \rightarrow X, \pi^{r, s}: J^{r} Y \rightarrow J^{s} Y$, $0 \leq s \leq r$, are the canonical jet projections. For any open subset $W \subset Y$, we put $W^{r}=\left(\pi^{r, 0}\right)^{-1}(W)$, an open subset of $J^{r} Y$. An element $J_{x}^{r} \gamma$ of $J^{r} Y$ is the $r$-jet of section $\gamma$ of $Y$ with source $x \in X$ and target $y=\gamma(x)$. The mapping $x \rightarrow J^{r} \gamma(x)=J_{x}^{r} \gamma$ is the $r$-jet prolongation of $\gamma$. The set $J^{r} Y$ is considered with its natural fibered manifold structure: if $(V, \psi), \psi=\left(t, y^{\sigma}\right), 1 \leq \sigma \leq m$, is a fibered chart on $Y$, the associated chart on $J^{r} Y$ (resp. on $X$ ), reads $\left(V^{r}, \psi^{r}\right)$, $\psi^{r}=\left(t, y^{\sigma}, y_{1}^{\sigma}, y_{2}^{\sigma}, \ldots, y_{r}^{\sigma}\right)$, resp. $(U, \varphi), \varphi=(t)$, where $V^{r}=\left(\pi^{r, 0}\right)^{-1}(V)$, resp. $U=\pi(V)$. Recall that by definition of an $r$-jet $J_{x}^{r} \gamma \in V^{r}$ the coordinate $y_{l}^{\sigma}\left(J_{x}^{r} \gamma\right)$ is defined to be $D^{l}\left(y^{\sigma} \gamma \varphi^{-1}\right)(\varphi(x))$.

We denote by $\Omega_{0}^{r} W$ the ring of differentiable functions defined on $W^{r} \subset J^{r} Y$, and by $\Omega_{k}^{r} W$ the $\Omega_{0}^{r} W$-module of differentiable $k$-forms on $W^{r}$. The exterior algebra of differential forms on $W^{r}$ is denoted by $\Omega^{r} W$. For any function $f: W^{r} \rightarrow \mathbb{R}$, we put $h f=f \circ \pi^{r+1, r}$, and with the help of the chart formulas $h d t=d t, h d y_{l}^{\sigma}=y_{l+1}^{\sigma} d t$. These formulas define a (global) homomorphism of exterior algebras $h: \Omega^{r} W \rightarrow$ $\Omega^{r+1} W$ called the $\pi$-horizontalization. In particular, $h(d f)=(d f / d t) d t$, where $d f / d t$ is the formal derivative of function $f$.

A 1-form $\rho \in \Omega_{1}^{r} W$ is said to be contact, if $h \rho=0$ or, equivalently, if $\left(J^{r} \gamma\right)^{*} \rho$ vanishes for any section $\gamma$ of $\pi$, defined on an open subset of $X$. In arbitrary fibered chart $(V, \psi), \psi=\left(t, y^{\sigma}\right)$, on $W \subset Y$, every contact 1-form $\rho$ has an expression $\rho=A_{\sigma}^{l} \omega_{l}^{\sigma}$ (sum through $0 \leq l \leq r-1,1 \leq \sigma \leq m$ ) for some functions $A_{\sigma}^{l}: V^{r} \rightarrow \mathbb{R}$, where $\omega_{l}^{\sigma}=d y_{l}^{\sigma}-y_{l+1}^{\sigma} d t$. A basis of 1-forms on $V^{r}$, constituted by the forms $d t$, $\omega_{l}^{\sigma}, d y_{r}^{\sigma}$, where $0 \leq l \leq r-1$, is called the contact basis. If $(\bar{V}, \bar{\psi}), \bar{\psi}=\left(\bar{t}, \bar{y}^{\nu}\right)$, is another fibered chart on $W \subset Y$ such that $V \cap \bar{V} \neq \emptyset$, then the contact 1-forms obey the transformation property $\bar{\omega}_{l}^{\nu}=\sum_{s=0}^{l}\left(\partial \bar{y}_{l}^{\nu} / \partial y_{s}^{\sigma}\right) \omega_{s}^{\sigma}$. This means, in particular, that contact 1 -forms $\omega_{l}^{\sigma}$ locally generate an ideal in the exterior algebra $\Omega^{r} W$ of differential forms on $W^{r}$, called the contact ideal.

Let $\rho$ be a differential 1-form on $W^{r} \subset J^{r} Y$. The pull-back of $\rho$ with respect to the jet projection $\pi^{r+1, r}: J^{r+1} Y \rightarrow J^{r} Y$ has a unique decomposition,

$$
\left(\pi^{r+1, r}\right)^{*} \rho=h \rho+p \rho
$$

where $h \rho$ (resp. $p \rho$ ) is a $\pi^{r+1}$-horizontal (resp. contact) 1-form on $W^{r+1}$. Indeed, if $\rho \in \Omega_{1}^{r} W$ is expressed by $\rho=A d t+B_{\sigma}^{l} d y_{l}^{\sigma}$, then it is easily seen that

$$
h \rho=\left(A+\sum_{l=0}^{r} B_{\sigma}^{l} y_{l+1}^{\sigma}\right) d t, \quad p \rho=\sum_{l=0}^{r} B_{\sigma}^{l} \omega_{l}^{\sigma} .
$$

Decomposition (11) can be directly generalized for arbitrary $k$-forms $\rho \in \Omega_{k}^{r} W$. We get

$$
\left(\pi^{r+1, r}\right)^{*} \rho=p_{k-1} \rho+p_{k} \rho
$$


where $p_{k-1} \rho$, resp. $p_{k} \rho$, is the $(k-1)$-contact, resp. $k$-contact component of $\rho$. This formula is the canonical decomposition of $\rho$. If $\rho=\kappa \wedge d t+\chi$, where

$$
\begin{aligned}
& \kappa=\frac{1}{(k-1) !} \sum_{l_{1}, l_{2}, \ldots, l_{k-1}=0}^{r} A_{\sigma_{1} \sigma_{2} \ldots \sigma_{k-1}}^{l_{1} l_{2} \ldots l_{k-1}} d y_{l_{1}}^{\sigma_{1}} \wedge d y_{l_{2}}^{\sigma_{2}} \wedge \ldots \wedge d y_{l_{k-1}}^{\sigma_{k-1}}, \\
& \chi=\frac{1}{k !} \sum_{l_{1}, l_{2}, \ldots, l_{k}=0}^{r} B_{\sigma_{1} \sigma_{2} \ldots \sigma_{k}}^{l_{1} l_{2} \ldots l_{k}} d y_{l_{1}}^{\sigma_{1}} \wedge d y_{l_{2}}^{\sigma_{2}} \wedge \ldots \wedge d y_{l_{k}}^{\sigma_{k}},
\end{aligned}
$$

then

$$
\begin{aligned}
p_{k-1} \rho & =\frac{1}{(k-1) !} \sum_{l_{1}, l_{2}, \ldots, l_{k}=0}^{r}\left(A_{\sigma_{1} \sigma_{2} \ldots \sigma_{k-1}}^{l_{1} l_{2} \ldots l_{k-1}}+B_{\sigma_{1} \sigma_{2} \ldots \sigma_{k}}^{l_{1} l_{2} \ldots l_{k}} y_{l_{k}+1}^{\sigma_{k}}\right) \\
& \cdot \omega_{l_{1}}^{\sigma_{1}} \wedge \omega_{l_{2}}^{\sigma_{2}} \wedge \ldots \wedge \omega_{l_{k-1}}^{\sigma_{k-1}} \wedge d t, \\
p_{k} \rho & =\frac{1}{k !} \sum_{l_{1}, l_{2}, \ldots, l_{k}=0}^{r} B_{\sigma_{1} \sigma_{2} \ldots \sigma_{k}}^{l_{1} l_{2} \ldots l_{k}} \omega_{l_{1}}^{\sigma_{1}} \wedge \omega_{l_{2}}^{\sigma_{2}} \wedge \ldots \wedge \omega_{l_{k}}^{\sigma_{k}} .
\end{aligned}
$$

A $k$-form $\rho \in \Omega_{k}^{r} W$ is said to be $k$-contact, if it coincides, up to the pull-back, with its $k$-contact component, that is, if $\left(\pi^{r+1, r}\right)^{*} \rho=p_{k} \rho$; similarly, $\rho$ is called $(k-1)$-contact, if $\left(\pi^{r+1, r}\right)^{*} \rho=p_{k-1} \rho$. The transformation property of contact 1-forms implies that $k$-contact, resp. $(k-1)$-contact, forms on $W^{r}$ constitute a submodule of the module $\Omega_{k}^{r} W$, which we denote by $\Omega_{k, c}^{r} W$, resp. $\Omega_{k-1, c}^{r} W$.

Let us remark that every $k$-form is contact for $k \geq 2$. We extend the notion of contactness for differential forms of arbitrary degree as follows. A $k$-form $\rho \in \Omega_{k}^{r} W$ is said to be strongly contact, if at every point of $Y$ there exist a fibered chart $(V, \psi)$, $\psi=\left(t, y^{\sigma}\right)$, and a $(k-1)$-contact $(k-1)$-form $\eta$ on $V^{r}$ such that

$$
p_{k}(\rho-d \eta)=0
$$

holds on $V^{r}$. This definition is also motivated by the construction of a variational sequence (cf. Sec. 3). It is easily seen that a $k$-form $\rho \in \Omega_{k}^{r} W$ is strongly contact if and only if at every point of $Y$ there exist a fibered chart $(V, \psi), \psi=\left(t, y^{\sigma}\right)$, a $k$-contact $k$-form $\mu$, and a $(k-1)$-contact $(k-1)$-form $\eta$, defined on $V^{r}$, such that $\rho=\mu+d \eta$. This formula defines a direct sum decomposition over the chart neighborhood $V$,

$$
\Omega_{k}^{r} V \ni \rho \rightarrow(\mu, \eta) \in \Omega_{k, c}^{r} V \oplus \Omega_{k-1, c}^{r} V .
$$

The exterior derivative of a strongly contact form is again strongly contact.

Now we shall consider smooth embeddings into Euclidean spaces which are morphisms of fibered manifolds over one-dimensional basis $\mathbb{R}$, and study basic properties of contact forms induced on embedded submanifolds. The canonical fibered coordinates on $\mathbb{R}^{m+1}=\mathbb{R} \times \mathbb{R}^{m}$ over $\mathbb{R}$ are denoted by $\left(t, x^{\sigma}\right), 1 \leq \sigma \leq m$, and $\left(t, x^{\sigma}, x_{1}^{\sigma}, x_{2}^{\sigma}, \ldots, x_{r}^{\sigma}\right)$ are the global associated coordinates on $J^{r}\left(\mathbb{R} \times \mathbb{R}^{m}\right)$. As usual, for lower orders we denote the derivative coordinates by the dot sign: if $r=2$, we write $\left(t, x^{\sigma}, \dot{x}^{\sigma}, \ddot{x}^{\sigma}\right)$ instead of $\left(t, x^{\sigma}, x_{1}^{\sigma}, x_{2}^{\sigma}\right)$.

Let $m, n$ be positive integers, $n \leq m$, and let $Q$ be an $n$-dimensional embedded submanifold of the Euclidean space $\mathbb{R}^{m}$. The canonical embedding

$$
\iota: \mathbb{R} \times Q \rightarrow \mathbb{R} \times \mathbb{R}^{m}
$$

is a smooth morphism of fibered manifolds over the identity mapping of the real line $\mathbb{R}$. The canonical projections $\pi_{Q}: \mathbb{R} \times Q \rightarrow \mathbb{R}$ and $\pi: \mathbb{R} \times \mathbb{R}^{m} \rightarrow \mathbb{R}$ satisfy $\pi \circ \iota=\pi_{Q}$, and every section $\gamma_{Q}$ of $\pi_{Q}$ induces a section $\gamma$ of $\pi$, defined by the composition 
$\gamma=\iota \circ \gamma_{Q}$. Indeed, for a section $\gamma_{Q}$ of $\pi_{Q}$ we have $\pi \circ \gamma=\pi \circ \iota \circ \gamma_{Q}=\pi_{Q} \circ \gamma_{Q}=\mathrm{id} \mathbb{R}$. Thus, we may define the $r$-th jet prolongation $J^{r} \iota$ of $\iota$ by the formula

$$
J^{r}(\mathbb{R} \times Q) \ni J_{t}^{r} \gamma_{Q} \rightarrow J^{r} \iota\left(J_{t}^{r} \gamma_{Q}\right)=J_{t}^{r}\left(\iota \circ \gamma_{Q}\right) \in J^{r}\left(\mathbb{R} \times \mathbb{R}^{m}\right) .
$$

Mapping (4) acts on differential forms by means of the pull-back operation. For arbitrary $k \geq 0$, we get a homomorphism of modules of differential forms,

$$
J^{r} \iota^{*}: \Omega_{k}^{r}\left(\mathbb{R} \times \mathbb{R}^{m}\right) \rightarrow \Omega_{k}^{r}(\mathbb{R} \times Q) .
$$

The following result is a straightforward application of the existence of partition of unity subordinate to an adapted atlas to a given embedded submanifold. In particular, it shows that the pull-back (5) is a surjective mapping.

Lemma 1. Let $Q$ be an embedded submanifold of $\mathbb{R}^{m}, W \subset \mathbb{R} \times Q$ an open set. Suppose $\eta \in \Omega_{k}^{r} W$ be a $k$-form on $W^{r} \subset J^{r}(\mathbb{R} \times Q)$. Then there exist a neighbourhood $U$ of $\iota(W)$ in $\mathbb{R}^{m+1}$ and a $k$-form $\tilde{\eta} \in \Omega_{k}^{r} U$ on $U^{r} \subset J^{r} \mathbb{R}^{m+1}$ such that $J^{r} \iota^{*} \tilde{\eta}=\eta$.

Remark 1 . Note that Lemma 1 holds also for contact and strongly contact forms. We shall utilize this lemma in the study of variational sequences (Sec. 3.2).

For further considerations we find a chart expression of $J^{r} \iota$ (4) by a straightforward calculation.

Lemma 2. Let $(U, \varphi), \varphi=\left(t, q^{i}\right), 1 \leq i \leq n$, be a fibered chart on $\mathbb{R} \times Q$. Suppose the embedding $\iota: \mathbb{R} \times Q \rightarrow \mathbb{R} \times \mathbb{R}^{m}$ is expressed by equations

$$
t \circ \iota=t, \quad x^{\sigma} \circ \iota=f^{\sigma}\left(q^{i}\right), \quad 1 \leq i \leq n, 1 \leq \sigma \leq m .
$$

Then the $r$-th prolongation $J^{r} \iota$ of $\iota$ is expressed in the associated chart $\left(U^{r}, \varphi^{r}\right)$, $\varphi^{r}=\left(t, q^{i}, q_{1}^{i}, q_{2}^{i}, \ldots, q_{r}^{i}\right)$, on $J^{r}(\mathbb{R} \times Q)$ by the equations

$$
\begin{aligned}
& t \circ J^{r} \iota=t, \quad x^{\sigma} \circ J^{r} \iota=f^{\sigma}\left(q^{i}\right), \quad \dot{x}^{\sigma} \circ J^{r} \iota=\frac{d}{d t} f^{\sigma}\left(q^{i}\right), \\
& \ddot{x}^{\sigma} \circ J^{r} \iota=\frac{d^{2}}{d t^{2}} f^{\sigma}\left(q^{i}\right), \quad x_{l}^{\sigma} \circ J^{r} \iota=\frac{d^{l}}{d t^{l}} f^{\sigma}\left(q^{i}\right),
\end{aligned}
$$

where $0 \leq l \leq r$.

The following lemma describes properties of contact forms with respect to the pull-back $J^{r} \iota^{*}$ (5). In particular, we show that the property of contactness and strongly contactness is preserved along a jet prolongation of an embedding.

Lemma 3. (a) If a 1-form $\rho \in \Omega_{1}^{r} R^{m+1}$ is contact, then $J^{r} \iota^{*} \rho \in \Omega_{1}^{r}(\mathbb{R} \times Q)$ is contact.

(b) The pull-back $J^{r} \iota^{*} \rho$ of any strongly contact $k$-form $\rho$ on $J^{r} \mathbb{R}^{m+1}$ is strongly contact.

(c) Suppose the embedding $\iota: \mathbb{R} \times Q \rightarrow \mathbb{R}^{m+1}$ is expressed with respect to a fibered chart $(U, \varphi), \varphi=\left(t, q^{i}\right)$, on $S$ by equations (6). Then pull-backs of contact 1-forms 
$\omega_{l}^{\sigma}, 0 \leq l \leq r-1$, on $J^{r} \mathbb{R}^{m+1}$ with respect to $J^{r} \iota$ read

$$
\begin{aligned}
J^{r} \iota^{*} \omega^{\sigma} & =\frac{\partial f^{\sigma}}{\partial q^{i}} \eta^{i}, \\
J^{r} \iota^{*} \dot{\omega}^{\sigma} & =\frac{d}{d t}\left(\frac{\partial f^{\sigma}}{\partial q^{i}}\right) \eta^{i}+\frac{\partial f^{\sigma}}{\partial q^{i}} \dot{\eta}^{i}, \\
J^{r} \iota^{*} \ddot{\omega}^{\sigma} & =\frac{d^{2}}{d t^{2}}\left(\frac{\partial f^{\sigma}}{\partial q^{i}}\right) \eta^{i}+2 \frac{d}{d t}\left(\frac{\partial f^{\sigma}}{\partial q^{i}}\right) \dot{\eta}^{i}+\frac{\partial f^{\sigma}}{\partial q^{i}} \ddot{\eta}^{i}, \\
J^{r} \iota^{*} \omega_{l}^{\sigma} & =\sum_{s=0}^{l} \frac{\partial}{\partial q_{s}^{i}}\left(\frac{d^{l} f^{\sigma}}{d t^{l}}\right) \eta_{s}^{i}, \quad 0 \leq l \leq r-1,
\end{aligned}
$$

where $\eta_{s}^{i}=d q_{s}^{i}-q_{s+1}^{i} d t, 0 \leq s \leq r-1,1 \leq i \leq n$, are contact forms on $U^{r} \subset$ $J^{r}(\mathbb{R} \times Q)$.

Proof. (a) Suppose $\rho$ is a contact 1 -form on $J^{r} \mathbb{R}^{m+1}$, that is by definition, the pull-back $J^{r} \gamma^{*} \rho$ vanishes for every smooth section $\gamma$ of $\pi: \mathbb{R}^{m+1} \rightarrow \mathbb{R}$. Let $\gamma_{Q}$ be a section of $\pi_{Q}: \mathbb{R} \times Q \rightarrow \mathbb{R}$. We compute the pull-back $J^{r} \gamma_{Q}{ }^{*} J^{r} \iota^{*} \rho$. For every point $t$ from the domain of $\gamma_{Q},\left(J^{r} \iota \circ J^{r} \gamma_{Q}\right)(t)=J^{r} \iota\left(J_{t}^{r} \gamma_{Q}\right)=J_{t}^{r}\left(\iota \circ \gamma_{Q}\right)=J^{r}\left(\iota \circ \gamma_{Q}\right)(t)$, hence we get

$$
J^{r} \gamma_{Q}{ }^{*} J^{r} \iota^{*} \rho=\left(J^{r} \iota \circ J^{r} \gamma_{Q}\right)^{*} \rho=J^{r}\left(\iota \circ \gamma_{Q}\right)^{*} \rho .
$$

Since $\iota \circ \gamma_{Q}$ is a section of $\mathbb{R}^{m+1}$, by the assumption on contactness of $\rho$ this expression vanishes hence 1 -form $J^{r} \iota^{*} \rho$ on $J^{r}(\mathbb{R} \times Q)$ is a also contact, as required.

(b) For $k=1$ the assertion is equivalent with (a). Let $k \geq 2$, and suppose $\rho \in \Omega_{k}^{r} \mathbb{R}^{m+1}$ is decomposed as $\rho=\mu+d \eta$, where $\mu$, resp. $\eta$, is a $k$-contact $k$ form, resp. $(k-1)$-contact $(k-1)$-form. Then $J^{r} \iota^{*} \rho=J^{r} \iota^{*} \mu+d J^{r} \iota^{*} \eta$. Since a pull-back of wedge products equals wedge products of pull-backs, we obtain by the assertion (a) that also $J^{r} \iota^{*} \mu$, resp. $J^{r} \iota^{*} \eta$, is a $k$-contact $k$-form, resp. $(k-1)$ contact $(k-1)$-form, on $J^{r}(\mathbb{R} \times Q)$. This means, however, that $J^{r} \iota^{*} \rho \in \Omega_{k}^{r}(\mathbb{R} \times Q)$ is strongly contact.

(c) Formulas (8) are obtained by a staighforward calculation.

Remark 2 (Adapted charts). In order to simplify calculations and proofs, we may take advantage of adapted charts to submanifolds. Let $Q$ be an embedded $n$ dimensional submanifold of $Y$, where $\operatorname{dim} Y=m, n \leq m$. Recall that a chart $(V, \psi)$, $\psi=\left(u^{\sigma}\right)$, on $Y$ is adapted to $Q$, if there exist constants $c^{n+1}, c^{n+2}, \ldots, c^{m} \in \mathbb{R}$ such that the set $Q$ is expressed in $(V, \psi)$ by equations $u^{\kappa} \circ \iota=c^{\kappa}, n+1 \leq \kappa \leq m$, where $\iota: Q \rightarrow Y$ is the canonical smooth embedding. In this case, the set $Q \cap V$ is also called an $n$-dimensional slice in an open subset $V$ of $Y$. Without loss of generality, we may restrict ourselves to adapted charts to submanifold $Q$ which are rectangle hence the canonical embedding $\iota$ has equations

$$
u^{j} \circ \iota=\left.u^{j}\right|_{V \cap Q}, 1 \leq j \leq n, \quad u^{\kappa} \circ \iota=0, n+1 \leq \kappa \leq m .
$$

If $(V, \psi), \psi=\left(u^{\sigma}\right)$, is a chart on $\mathbb{R}^{m}$, adapted to $n$-dimensional submanifold $Q$ of $\mathbb{R}^{m}$, and satisfying (9), one can easily observe that the chart expressions (7) of Lemma 2, resp. (8) of Lemma 3, with respect to the associated chart on $Q$ reduce to non-trivial expressions for $q_{l}^{j} \circ J^{r} \iota$, resp. $J^{r} \iota^{*} \omega_{l}^{j}$, only. 


\section{VARIATIONAL SEQUENCES}

3.1. Variational sequences over fibered manifolds. Let us recall basic results. Suppose $Y$ to be a fibered manifold over a one-dimensional base $X$, where $\operatorname{dim} Y=$ $m+1, m \geq 0$. Let $k \geq 0, r \geq 0$, be integers. Consider the sheaf of smooth $k$-forms over $J^{r} Y$ and its direct image with respect to the jet projection $\pi^{r, 0}: J^{r} Y \rightarrow Y$, denoted by $\Omega_{k}^{r}$. Similarly, $\Omega_{k, c}^{r}$ (resp. $\Omega_{k-1, c}^{r}$ ) is the direct image of the sheaf of $k$-contact $k$-forms (resp. $(k-1)$-contact $(k-1)$-forms) over $J^{r} Y$ with respect to $\pi^{r, 0}$. We let denote $\Omega_{0, c}^{r}=\{0\}, \Omega_{k, c}^{r}=\operatorname{Ker} p_{k-1}$, and $\Theta_{k}^{r}=\Omega_{k, c}^{r}+d \Omega_{k-1, c}^{r}, k \geq 1$, where $p_{k-1}: \Omega_{k}^{r} \rightarrow \Omega_{k-1, c}^{r+1}$ is the sheaf space morphism assigning to a $k$-form its $(k-1)$-contact component (see Sec. 2, (21)), and $d \Omega_{k-1, c}^{r}$ is the image sheaf of $\Omega_{k-1, c}^{r}$ with respect to the exterior derivative morphism $d$.

For arbitrary open subset $W$ of $Y$, we have $\Omega_{k}^{r} W\left(\right.$ resp. $\Omega_{k, c}^{r} W$ ) the Abelian group of differential $k$-forms (resp. $k$-contact $k$-forms), defined on an open subset $W^{r}=\left(\pi^{r, 0}\right)^{-1}(W)$ of the $r$-th jet prolongation $J^{r} Y . d \Omega_{k-1, c}^{r} W$ is the Abelian group of $k$-forms on $W^{r}$, locally expressed as exterior derivatives of $(k-1)$-contact $(k-1)$-forms, and $\Theta_{k}^{r} W$ is a subgroup (but not a submodule) of $\Omega_{k}^{r} W$, elements of which are the strongly contact $k$-forms on $W^{r}$ (see Sec. 2, (3)).

From decomposition properties of strongly contact forms, we observe that the exterior derivative operator $d$ defines a subsequence

$$
0 \rightarrow \Theta_{1}^{r} \rightarrow \Theta_{2}^{r} \rightarrow \cdots \rightarrow \Theta_{M}^{r} \rightarrow 0
$$

of the de Rham sequence $0 \rightarrow \mathbb{R} \rightarrow \Omega_{0}^{r} \rightarrow \Omega_{1}^{r} \rightarrow \Omega_{2}^{r} \rightarrow \ldots \rightarrow \Omega_{N}^{r} \rightarrow 0$, where $M=m r+1, N=\operatorname{dim} J^{r} Y=m(r+1)+1$. (10) is the contact subsequence, and the quotient sequence

$$
\begin{aligned}
0 \rightarrow & \mathbb{R}_{Y} \rightarrow \Omega_{0}^{r} \rightarrow \Omega_{1}^{r} / \Theta_{1}^{r} \rightarrow \Omega_{2}^{r} / \Theta_{2}^{r} \rightarrow \cdots \\
& \rightarrow \Omega_{M}^{r} / \Theta_{M}^{r} \rightarrow \Omega_{M+1}^{r} \rightarrow \cdots \rightarrow \Omega_{N}^{r} \rightarrow 0,
\end{aligned}
$$

briefly denoted by $0 \rightarrow \mathbb{R}_{Y} \rightarrow \mathcal{V}_{Y}^{r}$, is the variational sequence of order $r$ over $Y$. An element of $\Omega_{k}^{r} / \Theta_{k}^{r}$, represented by a $k$-form $\rho \in \Omega_{k}^{r} W$, is denoted by $[\rho]$. Morphisms $E_{k}: \Omega_{k}^{r} / \Theta_{k}^{r} \rightarrow \Omega_{k+1}^{r} / \Theta_{k+1}^{r}$ in (11) are defined by

$$
E_{k}([\rho])=[d \rho] .
$$

Summarizing, we have the commutative diagram

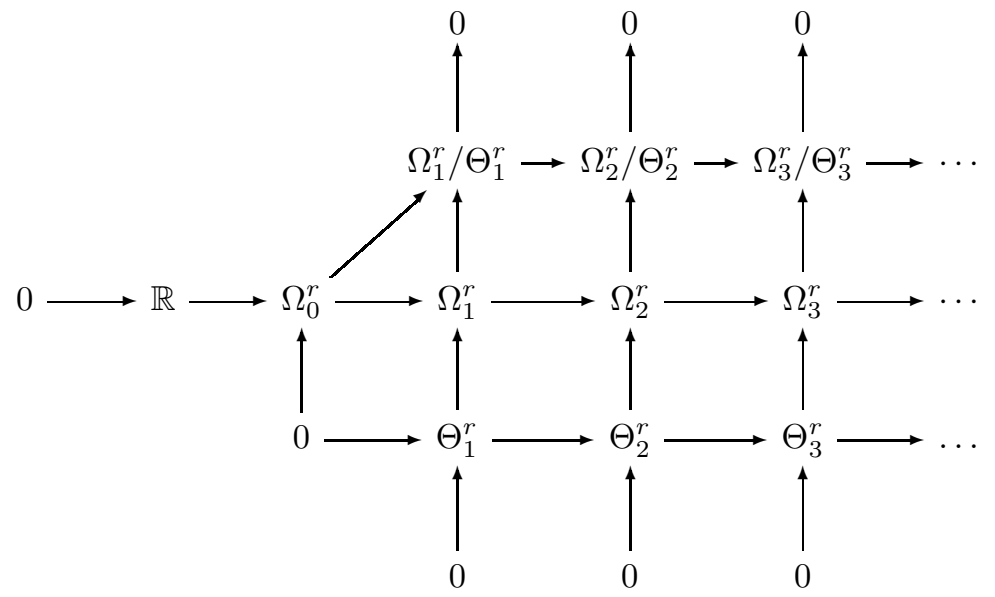

Now we describe fundamental properties of the variational sequence theory. 
Theorem 1. The contact subsequence (10) is exact.

Since exactness of a quotient sequence follows from exactness of its subsequence of Abelian groups, Theorem 1 immediately implies that the variational sequence is also exact hence $0 \rightarrow \mathbb{R}_{Y} \rightarrow \mathcal{V}_{Y}^{r}$ (11) constitutes a resolution of the constant sheaf $\mathbb{R}_{Y}$.

The next result plays an important role in the study of order reduction of variational objects, embeddings of $r$-th order variational sequences into sequences of order $r+1$, and it serves as the main tool for finding suitable local representatives of classes in the variational sequence. Since the quotient groups are determined up to an isomorphism, we are allowed to consider the classes in the variational sequence (11) as elements of different sheaves.

Theorem 2. The quotient mapping $\Omega_{k}^{r} W / \Theta_{k}^{r} W \rightarrow \Omega_{k}^{r+1} W / \Theta_{k}^{r+1} W$, corresponding to the pull-back $\Omega_{k}^{r} W \ni \rho \rightarrow\left(\pi^{r+1, r}\right)^{*} \rho \in \Omega_{k}^{r+1} W$, is injective.

Recall that a sheaf $S$ over a topological space $X$ is called soft, if every continuous section of $S$, defined on a closed subset of $X$, can be prolonged to a global continuous section, and a sheaf $S$ over $X$ is called fine, if to every locally finite open covering of $X$ there exists a subordinate sheaf partition of unity of the sheaf space germ $S$. Every fine sheaf over a paracompact Hausdorff space is soft.

Theorem 3. Every of the sheaves $\Omega_{k}^{r}$ is fine, and hence soft.

Now, as a consequence of Theorem 3 , we get a theorem on global properties of the variational sequence. Recall that a resolution of an Abelian sheaf $S$ over $X$, $0 \rightarrow S \rightarrow S^{0} \rightarrow S^{1} \rightarrow S^{2} \rightarrow \ldots$, is called acyclic, if $H^{k}\left(X, S^{i}\right)=0$ for all $i \geq 1$. In particular, every soft sheaf $S$ over $X$ satisfies $H^{k}(X, S)=0, k \geq 1$.

Theorem 4. The variational sequence of order $r$ over $Y$ (11) is an acyclic resolution of the constant sheaf $\mathbb{R}_{Y}$ over $Y$.

Denote by $\Gamma \mathcal{V}_{Y}^{r}$ the cochain complex of Abelian groups of global sections,

$$
\begin{aligned}
0 & \rightarrow \Gamma\left(Y, R_{Y}\right) \rightarrow \Gamma\left(Y, \Omega_{0}^{r}\right) \rightarrow \Gamma\left(Y, \Omega_{1}^{r} / \Theta_{1}^{r}\right) \rightarrow \Gamma\left(Y, \Omega_{2}^{r} / \Theta_{2}^{r}\right) \\
& \rightarrow \cdots \rightarrow \Gamma\left(Y, \Omega_{M}^{r} / \Theta_{M}^{r}\right) \rightarrow \Gamma\left(Y, \Omega_{M+1}^{r}\right) \rightarrow \cdots \rightarrow \Gamma\left(Y, \Omega_{N}^{r}\right) \rightarrow 0,
\end{aligned}
$$

and by $H^{k}\left(\Gamma \mathcal{V}_{Y}^{r}\right)$ the $k$-th cohomological group of (13), this means $H^{k}\left(\Gamma \mathcal{V}_{Y}^{r}\right)=$ Ker $E_{k} / \operatorname{Im} E_{k-1}$. The following result is a consequence of Theorem 4 and the well-known abstract de Rham theorem (cf. Warner [16]).

Theorem 5. The cohomology of the complex (13) and the de Rham cohomology of the underlying manifold $Y$ coincide, i.e. for every $k \geq 0$

$$
H^{k}\left(\Gamma \mathcal{V}_{Y}^{r}\right)=H_{\mathrm{deR}}^{k} Y .
$$

3.2. Variational sequences induced by embedded submanifolds. Let $Q$ be an embedded submanifold of $\mathbb{R}^{m}$, and $\iota: \mathbb{R} \times Q \rightarrow \mathbb{R} \times \mathbb{R}^{m}$ be the canonical embedding of fibered manifolds over $\mathbb{R}$, locally expressed by equations $t \circ \iota=t$, $x^{\sigma} \circ \iota=f^{\sigma}\left(q^{i}\right)$ (Sec. 2, (6) ), with respect to the global canonical coordinates $\left(t, x^{\sigma}\right)$ on $\mathbb{R} \times \mathbb{R}^{m}$, and a fibered chart $(U, \varphi), \varphi=\left(t, q^{i}\right)$, on $\mathbb{R} \times Q$. The $r$-th jet prolongation $J^{r} \iota: J^{r}(\mathbb{R} \times Q) \rightarrow J^{r}\left(\mathbb{R} \times \mathbb{R}^{m}\right)$ (Sec. 2, (4)) has a chart expression described by Lemma 2 .

Let us study variational sequences over both fibered manifolds $\mathbb{R} \times Q$ and $\mathbb{R} \times \mathbb{R}^{m}$. To this purpose we introduce a pull-back of presheaves of forms with respect to an 
embedding. Denote by $\Omega_{k}^{r}$, resp. $Q_{\Omega}^{r}$, the direct image of the sheaf of smooth $k$-forms over $J^{r}\left(\mathbb{R} \times \mathbb{R}^{m}\right)$, resp. $J^{r}(\mathbb{R} \times Q)$, with respect to the jet projection $\pi^{r, 0}: J^{r}\left(\mathbb{R} \times \mathbb{R}^{m}\right) \rightarrow \mathbb{R} \times \mathbb{R}^{m}$, resp. $\pi_{Q}^{r, 0}: J^{r}(\mathbb{R} \times Q) \rightarrow \mathbb{R} \times Q$. It should be pointed out that $\Omega_{k}^{r}$ is a simplified notation for the sheaf $\mathbb{R}^{m} \Omega_{k}^{r}$. Analogously to Sec. 3.1. we denote the sheaves of strongly contact forms by $\Theta_{k}^{r}=\Omega_{k, c}^{r}+d \Omega_{k-1, c}^{r}$, and $Q_{\Theta}^{r}=Q_{\Omega_{k, c}^{r}}+d^{Q} \Omega_{k-1, c}^{r}$. An element of $\Omega_{k}^{r} / \Theta_{k}^{r}$, represented by a $k$-form $\rho \in \Omega_{k}^{r} U$, is denoted by $[\rho]$; an element of $Q_{\Omega_{k}^{r}} / Q^{Q} \Theta_{k}^{r}$, represented by a $k$-form $\tau \in Q_{\Omega_{k}^{r}} W$, is denoted by $[\tau]_{Q}$.

The following constructions allow us to introduce variational sequences on embedded submanifolds. For arbitrary open set $W \subset \mathbb{R} \times Q$ we put

and

$$
\begin{aligned}
& J^{r} \iota^{*}\left(\Omega_{k}^{r}\right)(W)=\left\{\left.J^{r} \iota^{*} \rho\right|_{W^{r}} \mid \rho \in \Omega_{k}^{r} U\right\}, \\
& J^{r} \iota^{*}\left(\Theta_{k}^{r}\right)(W)=\left\{\left.J^{r} \iota^{*} \rho\right|_{W^{r}} \mid \rho \in \Theta_{k}^{r} U\right\},
\end{aligned}
$$

where $U$ runs over neighbourhoods of $\iota(W)$. From definition (15) it is straightforward that the correspondence assigning to an open set $W \subset \mathbb{R} \times Q$ the Abelian group $J^{r} \iota^{*}\left(\Omega_{k}^{r}\right)(W)$, resp. $J^{r} \iota^{*}\left(\Theta_{k}^{r}\right)(W)$, is a presheaf, and $J^{r} \iota^{*}\left(\Omega_{k}^{r}\right)(W)$, resp. $J^{r} \iota^{*}\left(\Theta_{k}^{r}\right)(W)$, is an Abelian subgroup of ${ }^{Q} \Omega_{k}^{r} W$, resp. ${ }^{Q} \Theta_{k}^{r} W$, for every open set $W$. The next lemma describes the presheaf (16).

Theorem 6. The presheaf $J^{r} \iota^{*}\left(\Omega_{k}^{r} / \Theta_{k}^{r}\right)$ is a sheaf, and it coincides with the quotient sheaf ${ }^{Q} \Omega_{k}^{r} /{ }^{Q} \Theta_{k}^{r}$,

$$
J^{r} \iota^{*}\left(\Omega_{k}^{r} / \Theta_{k}^{r}\right)=Q_{\Omega_{k}^{r}} /{ }^{Q} \Theta_{k}^{r} .
$$

Proof. By Lemma 1 we have $J^{r} \iota^{*}\left(\Omega_{k}^{r}\right)(W)=Q_{\Omega_{k}^{r}} W, J^{r} \iota^{*}\left(\Theta_{k}^{r}\right)(W)=Q_{\Theta_{k}^{r}} W$, for every open set $W \subset \mathbb{R} \times Q$, and thus $J^{r} \iota^{*}\left(\Omega_{k}^{r}\right)$, resp. $J^{r} \iota^{*}\left(\Theta_{k}^{r}\right)$, coincides with the sheaf $Q \Omega_{k}^{r}$, resp. ${ }^{Q} \Theta_{k}^{r}$. From (16), however, it follows that $J^{r} \iota^{*}\left(\Omega_{k}^{r} / \Theta_{k}^{r}\right)(W)$ is the quotient group $J^{r} \iota^{*}\left(\Omega_{k}^{r}\right)(W) / J^{r} \iota^{*}\left(\Theta_{k}^{r}\right)(W)$. Hence we obtain the required identity.

Consider a sheaf morphism $J^{r} \iota^{*}: \Omega_{k}^{r} / \Theta_{k}^{r} \rightarrow{ }^{Q} \Omega_{k}^{r} /{ }^{Q} \Theta_{k}^{r}$, defined as follows. For any element $[\rho]$ of $\Omega_{k}^{r} / \Theta_{k}^{r}$, where $\rho \in \Omega_{k}^{r} U, U$ is an open subset of $\mathbb{R}^{m+1}$, we put

$$
J^{r} \iota^{*}([\rho])=\left[J^{r} \iota^{*} \rho\right]_{Q} .
$$

It is easy to see that definition (17) does not depend on the choice of a representative $\rho$ of class the $[\rho]$. Indeed, if $\rho^{\prime}$ is another representative of $[\rho]$, then $\rho-\rho^{\prime}$ belongs to $\Theta_{k}^{r}$. However, by Lemma 3. (b), it follows that $J^{r} \iota^{*}\left(\rho-\rho^{\prime}\right) \in{ }^{Q} \Theta_{k}^{r}$ hence we get

$$
\left[J^{r} \iota^{*} \rho\right]_{Q}=\left[J^{r} \iota^{*}\left(\rho-\rho^{\prime}\right)\right]_{Q}+\left[J^{r} \iota^{*} \eta\right]_{Q}=\left[J^{r} \iota^{*} \eta\right]_{Q}
$$

The variational sequence morphisms $E_{k}: \Omega_{k}^{r} / \Theta_{k}^{r} \rightarrow \Omega_{k+1}^{r} / \Theta_{k+1}^{r}$, and ${ }^{Q} E_{k}$ : ${ }^{Q} \Omega_{k}^{r} /{ }^{Q} \Theta_{k}^{r} \rightarrow{ }^{Q} \Omega_{k+1}^{r} /{ }^{Q} \Theta_{k+1}^{r}$, are defined by $E_{k}([\rho])=[d \rho]$, and ${ }^{Q} E_{k}\left([\tau]_{Q}\right)=[d \tau]_{Q}$, respectively (Sec. [3.1, (12)). We have the diagram

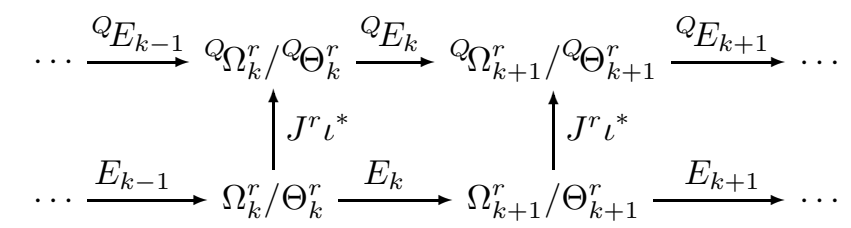

where by Lemma 6 the morphisms $J^{r} \iota^{*}$ (17) are surjective. The following theorem shows that squares in (18) commute. 
Theorem 7. For every positive integer $k$, the morphisms $E_{k}$ in the variational sequence $0 \rightarrow \mathbb{R}_{\mathbb{R}^{m}} \rightarrow \mathcal{V}_{\mathbb{R}^{m}}^{r}$ over $\mathbb{R} \times \mathbb{R}^{m}$, and ${ }^{Q} E_{k}$ in the variational sequence $0 \rightarrow \mathbb{R}_{Q} \rightarrow \mathcal{V}_{Q}^{r}$ over $\mathbb{R} \times Q$ satisfy the identity

$$
{ }^{Q} E_{k} \circ J^{r} \iota^{*}=J^{r} \iota^{*} \circ E_{k} .
$$

Proof. Identity (19) follows immediately from definitions. Indeed, applying formula (17) for $J^{r} \iota^{*}$ and the definition of morphisms $E_{k}$, we get for any element $\rho \in \Omega_{k}^{r} U$,

$$
\begin{aligned}
\left({ }^{Q} E_{k} \circ J^{r} \iota^{*}\right)([\rho]) & ={ }^{Q} E_{k}\left(\left[J^{r} \iota^{*} \rho\right]_{Q}\right)=\left[d J^{r} \iota^{*} \rho\right]_{Q}=\left[J^{r} \iota^{*} d \rho\right]_{Q} \\
& =J^{r} \iota^{*}([d \rho])=\left(J^{r} \iota^{*} \circ E_{k}\right)([\rho]),
\end{aligned}
$$

as required.

Remark 3. Theorems 6 and 7 say that the variational sequence $0 \rightarrow \mathbb{R}_{Q} \rightarrow \mathcal{V}_{Q}^{r}$ over $\mathbb{R} \times Q$ is completely described by the embedded submanifold $Q$ of $\mathbb{R}^{m}$ and the variational sequence $0 \rightarrow \mathbb{R}_{\mathbb{R}^{m}} \rightarrow \mathcal{V}_{\mathbb{R}^{m}}^{r}$ over $\mathbb{R} \times \mathbb{R}^{m}$.

Denote by $\Gamma \mathcal{V}_{\mathbb{R}^{m}}^{r}$ and $\Gamma \mathcal{V}_{Q}^{r}$, the cochain complexes of global sections associated with the variational sequences $0 \rightarrow \mathbb{R}_{\mathbb{R}^{m}} \rightarrow \mathcal{V}_{\mathbb{R}^{m}}^{r}$ and $0 \rightarrow \mathbb{R}_{Q} \rightarrow \mathcal{V}_{Q}^{r}$, respectively (cf. (13)). Theorem 7 allows us to introduce a cochain mapping, consisting of a family of homomorphisms $J^{r} \iota^{*}: \Gamma\left(\mathbb{R} \times \mathbb{R}^{m}, \Omega_{k}^{r} / \Theta_{k}^{r}\right) \rightarrow \Gamma\left(\mathbb{R} \times Q,{ }^{Q} \Omega_{k}^{r} /{ }^{Q} \Theta_{k}^{r}\right)$, defined by means of (17). The $k$-th cohomological group $H^{k}\left(\Gamma \mathcal{V}_{\mathbb{R}^{m}}^{r}\right)$, resp. $H^{k}\left(\Gamma \mathcal{V}_{Q}^{r}\right)$, of the complex $\Gamma \mathcal{V}_{\mathbb{R}^{m}}^{r}$, resp. $\Gamma \mathcal{V}_{Q}^{r}$, is defined in the standard sense (cf. Sec. 3.1). We get the induced homomorphism of cohomological groups $H_{\mathbb{R}^{m}, Q}^{k}: H^{k}\left(\Gamma \mathcal{V}_{\mathbb{R}^{m}}^{r}\right) \rightarrow H^{k}\left(\Gamma \mathcal{V}_{Q}^{r}\right)$, defined by $H_{\mathbb{R}^{m}, Q}^{k}([\rho])=\left[J^{r} \iota^{*} \rho\right]$ (cf. Warner [16]). The abstract de Rham theorem for cochain complexes of global sections of variational sequences, see Thereom [5] (14), implies that $H^{k}\left(\Gamma \mathcal{V}_{\mathbb{R}^{m}}^{r}\right)=H_{\mathrm{deR}}^{k}\left(\mathbb{R} \times \mathbb{R}^{m}\right)=0$ and $H^{k}\left(\Gamma \mathcal{V}_{Q}^{r}\right)=H_{\mathrm{deR}}^{k}(\mathbb{R} \times Q)=$ $H_{\mathrm{deR}}^{k} Q$, hence the homomorphism $H_{\mathbb{R}^{m}, Q}^{k}: 0 \rightarrow H_{\mathrm{deR}}^{k} Q$ is a trivial injection.

3.3. Applications: The calculus of variations. In this section we summarize basic higher-order variational formulas characterizing the Euler-Lagrange and Helmholtz mappings of the calculus of variations. One can easily specify these formulas for first- and second-order problems important for applications.

Let $(V, \psi), \psi=\left(t, y^{\sigma}\right)$, be a fibered chart on $W \subset Y$, and the associated chart on $W^{r} \subset J^{r} Y$ is denoted by $\left(V^{r}, \psi^{r}\right), \psi^{r}=\left(t, y^{\sigma}, y_{1}^{\sigma}, y_{2}^{\sigma}, \ldots, y_{r}^{\sigma}\right)$. If $\rho$ is a 1 -form on $W^{r} \subset J^{r} Y$, then its class $[\rho]$ is an element of $\Omega_{1}^{r} W / \Theta_{1}^{r} W$, represented by a differential 1-form $\lambda$ on $W^{r+1} \subset J^{r+1} Y$, with chart expression

$$
\lambda=\mathscr{L} d t
$$

where $\mathscr{L}: V^{r+1} \rightarrow \mathbb{R}$ is a real-valued function. $\lambda$ is the Lagrangian associated with $\rho$, and the function $\mathscr{L}$ is a (local) Lagrange function associated with $\lambda$.

If $\rho$ is a 2 -form on $W^{r}$, then its class $[\rho]$ is an element of $\Omega_{2}^{r} W / \Theta_{2}^{r} W$, represented by a differential 2-form $\varepsilon$ on $W^{s} \subset J^{s} Y$ for some $s \leq 2 r+1$, which has a chart expression

$$
\varepsilon=\varepsilon_{\sigma} \omega^{\sigma} \wedge d t
$$

where $\varepsilon_{\sigma}: V^{s} \rightarrow \mathbb{R}, \varepsilon_{\sigma}=\varepsilon_{\sigma}\left(t, y^{\sigma}, y_{1}^{\sigma}, y_{2}^{\sigma}, \ldots, y_{s}^{\sigma}\right)$. A differential 2-form locally expressed by (21) is a source form (of order $s$ ), associated with $\rho$. Every Lagrangian $\lambda$ defines a source form $E_{\lambda}$, locally expressed by

$$
E_{\lambda}=\mathcal{E}_{\sigma}(\mathscr{L}) \omega^{\sigma} \wedge d t
$$


where

$$
\mathcal{E}_{\sigma}(\mathscr{L})=\frac{\partial \mathscr{L}}{\partial y^{\sigma}}+\sum_{l=1}^{r}(-1)^{l} \frac{d^{l}}{d t^{l}} \frac{\partial \mathscr{L}}{\partial y_{l}^{\sigma}} .
$$

The coefficients of $E_{\lambda}$ are the Euler-Lagrange expressions, associated with $\mathscr{L}$. $E_{\lambda}$ is called the Euler-Lagrange form (or Euler-Lagrange class), associated with $\lambda$.

The class $[\rho]$ of a 3-form $\rho$ on $W^{r}$ belongs to the quotient $\Omega_{3}^{r} W / \Theta_{3}^{r} W$, and is represented by a differential 3-form $\vartheta$ on $W^{s} \subset J^{s} Y$ for some $s \leq 4 r+3$, which is locally expressed by

$$
\vartheta=\frac{1}{2} \sum_{l=0}^{r} \vartheta_{\nu \sigma}^{l} \omega_{l}^{\nu} \wedge \omega^{\sigma} \wedge d t
$$

where the components $\vartheta_{\nu \sigma}^{l}$ are functions of $t, y^{\kappa}, y_{1}^{\kappa}, y_{2}^{\kappa}, \ldots, y_{s}^{\kappa}$. Every form $\varepsilon$ (21) defines a class $H_{\varepsilon}$ in $\Omega_{3}^{r} W / \Theta_{3}^{r} W$ by

$$
H_{\varepsilon}=\mathcal{H}_{\nu \sigma}^{l}\left(\varepsilon_{\kappa}\right) \omega_{l}^{\nu} \wedge \omega^{\sigma} \wedge d t
$$

where

$$
\mathcal{H}_{\nu \sigma}^{l}\left(\varepsilon_{\kappa}\right)=\frac{\partial \varepsilon_{\sigma}}{\partial y_{l}^{\nu}}-(-1)^{l} \frac{\partial \varepsilon_{\nu}}{\partial y_{l}^{\sigma}}-\sum_{s=l+1}^{r}(-1)^{s}\left(\begin{array}{l}
s \\
l
\end{array}\right) \frac{d^{s-l}}{d t^{s-l}} \frac{\partial \varepsilon_{\nu}}{\partial y_{s}^{\sigma}}
$$

Then $H_{\varepsilon}$ is the Helmholtz form (Helmholtz class), associated with source form $\varepsilon$, and the components $\mathcal{H}_{\nu \sigma}^{l}\left(\varepsilon_{\kappa}\right)$ are the Helmholtz expressions.

The $\mathbb{R}$-linear mapping $\lambda \rightarrow E_{\lambda}$, assigning to a Lagrangian its Euler-Lagrange form, is the Euler-Lagrange mapping, and $\varepsilon \rightarrow H_{\varepsilon}$, assigning to a source form its Helmholtz form, we call the Helmholtz mapping. A Lagrangian $\lambda$ which belongs to the kernel of the Euler-Lagrange mapping, that is $E_{\lambda}=0$, is said to be (locally) trivial, or null. A source form $\varepsilon$ is said to be (locally) variational, if it belongs to the image of the Euler-Lagrange mapping; this means that there exists a Lagrangian $\lambda$ such that $\varepsilon=E_{\lambda}$, i.e. in components, $\varepsilon_{\sigma}$ coincide with the Euler-Lagrange expressions associated with a Lagrange function $\mathscr{L}$. In addition, if the Lagrangian $\lambda$ is defined on $J^{r+1} Y, \varepsilon$ is globally variational. The problem to describe the kernel and the image of the Euler-Lagrange mapping belongs to the inverse problem of the calculus of variations.

The following theorem explains the relationship between the variational sequence and the classical calculus of variations, and enables us to study global properties of the Euler-Lagrange and Helmholtz mappings.

Theorem 8. Let $W$ be an open subset in fibered manifold $Y$, and $(V, \psi), \psi=\left(t, q^{\sigma}\right)$, be a fibered chart on $Y$ such that $V \subset W$.

(a) Let $\lambda$ be a Lagrangian associated with a 1-form $\rho \in \Omega_{1}^{r} W$, given by (20). Then $E(\lambda)$ has an expression

$$
E(\lambda)=E_{\sigma}([d \rho]) \omega^{\sigma} \wedge d t
$$

where its coefficients coincide with the Euler-Lagrange expressions (23),

$$
E_{\sigma}([d \rho])=\mathcal{E}_{\sigma}(\mathscr{L}) .
$$


(b) Let $\varepsilon$ be a source form associated with a 2-form $\rho \in \Omega_{2}^{r} W$, given by (29). Then $E(\varepsilon)$ has an expression

$$
E(\varepsilon)=\frac{1}{2} \sum_{l=0}^{r} E_{\nu \sigma}^{l}([d \rho]) \omega_{l}^{\nu} \wedge \omega^{\sigma} \wedge d t
$$

where its coefficients coincide with the Helmholtz expressions (26),

$$
E_{\nu \sigma}^{l}([d \rho])=\mathcal{H}_{\nu \sigma}^{l}\left(\varepsilon_{\tau}\right)
$$

Corollary 1. (a) Let $\varepsilon$ be a source form on $J^{r} Y$. If $\varepsilon$ is locally variational and the second de Rham cohomology group of $Y$ vanishes, i.e. $H_{\mathrm{deR}}^{2} Y=0$, then $\varepsilon$ is globally variational.

(b) Let $\rho$ be a 1-form on $J^{r} Y$. If $\rho$ is locally variationally trivial and the first de Rham cohomology group of $Y$ vanishes, i.e. $H_{\mathrm{deR}}^{1} Y=0$, then $\rho$ is globally variationally trivial.

Consider now again the variational sequence $0 \rightarrow \mathbb{R}_{\mathbb{R}^{m}} \rightarrow \mathcal{V}_{\mathbb{R}^{m}}^{r}$ over the Euclidean space $\mathbb{R} \times \mathbb{R}^{m}$ and an embedded submanifold $Q$ of $\mathbb{R}^{m}$. As we observed, these data already determine the induced variational sequence $0 \rightarrow \mathbb{R}_{Q} \rightarrow \mathcal{V}_{Q}^{r}$ over $\mathbb{R} \times Q$. Since both $H_{\mathrm{deR}}^{2} \mathbb{R}^{m}$ and $H_{\mathrm{deR}}^{1} \mathbb{R}^{m}$ are trivial, we obtain by Corollary 1 that every locally variational source form on $J^{r}\left(\mathbb{R} \times \mathbb{R}^{m}\right)$ is automatically globally variational, and every locally variationally trivial 1 -form on $J^{r}\left(\mathbb{R} \times \mathbb{R}^{m}\right)$ is globally variationally trivial. In general, an analogous assertion is not valid when $\mathbb{R}^{m}$ is replaced with $Q$.

It is now straightforward to describe the local structure of the Euler-Lagrange and the Helmholtz forms in the induced variational sequence. In the following theorem we consider the embedded submanifold $Q$ and its equations expressed by (6), $t \circ \iota=t, x^{\sigma} \circ \iota=f^{\sigma}\left(q^{i}\right), 1 \leq i \leq n, 1 \leq \sigma \leq m . E_{\lambda}$ (resp. $\left.H_{\varepsilon}\right)$ is the Euler-Lagrange (resp. Helmholtz) form expressed by (22) (resp. (25)).

Theorem 9. (a) The Euler-Lagrange form $E_{J^{r} \iota^{*} \lambda}$, associated with $J^{r} \iota^{*} \lambda$, is expressed by

$$
E_{J^{r} \iota^{*} \lambda}=\left(\mathcal{E}_{\sigma}(\mathscr{L}) \circ J^{r} \iota\right) \frac{\partial f^{\sigma}}{\partial q^{i}} \eta^{i} \wedge d t
$$

where $\eta^{i}=d q^{i}-\dot{q}^{i} d$ t are contact 1 -forms on $U^{1} \subset J^{1}(\mathbb{R} \times Q)$.

(b) The Helmholtz form $H_{J^{r} \iota^{*} \varepsilon}$, associated with $J^{r} \iota^{*} \varepsilon$, is expressed by

$$
H_{J^{r} \iota^{*} \varepsilon}=\frac{1}{2} \sum_{l=0}^{r} \sum_{k=0}^{l}\left(H_{\sigma \nu}^{l}\left(\varepsilon_{\tau}\right) \circ J^{r} \iota\right) \frac{\partial f^{\nu}}{\partial q^{j}} \frac{\partial\left(x_{l}^{\sigma} \circ J^{r} \iota\right)}{\partial q_{k}^{i}} \eta_{k}^{i} \wedge \eta^{j} \wedge d t .
$$

Proof. By Theorem 7 (19), $E_{J^{r} \iota^{*} \lambda}={ }^{Q} E\left(J^{r} \iota^{*} \lambda\right)=J^{r} \iota^{*}\left(\mathbb{R}^{m} E(\lambda)\right)=J^{r} \iota^{*} E_{\lambda}$, and analogously $H_{J^{r} \iota^{*} \varepsilon}={ }^{Q} E\left(J^{r} \iota^{*} \varepsilon\right)=J^{r} \iota^{*}\left(\mathbb{R}^{m} E(\varepsilon)\right)=J^{r} \iota^{*} H_{\varepsilon}$. Applying now coordinate expressions for pull-backs with respect to $J^{r} \iota$ (Lemma 2 and 3), to the forms $J^{r} \iota^{*} E_{\lambda}$ and $J^{r} \iota^{*} H_{\varepsilon}$, we get the required expressions of $E_{J^{r} \iota^{*} \lambda}$ and $H_{J^{r} \iota^{*} \varepsilon}$.

We call (27) the $Q$-induced Euler-Lagrange form, associated with Lagrangian $\lambda$, and (28) the $Q$-induced Helmholtz form, associated with source form $\varepsilon$. 


\section{VARiational SUbmanifoldS of EuClidean SPaCES: SECOND-ORDER EQUATIONS}

An arbitrary system of second-order ordinary differential equations for an unknown section $t \rightarrow\left(t, x^{\nu}(t)\right)$ of $\mathbb{R} \times \mathbb{R}^{m}$,

$$
\varepsilon_{\sigma}\left(t, x^{\nu}, \dot{x}^{\nu}, \ddot{x}^{\nu}\right)=0
$$

where the coefficients $\varepsilon_{\sigma}, 1 \leq \sigma \leq m$, are differentiable functions, defines a source form on $J^{2}\left(\mathbb{R} \times \mathbb{R}^{m}\right)$ as

$$
\varepsilon=\varepsilon_{\sigma} \omega^{\sigma} \wedge d t
$$

where $\omega^{\sigma}=d x^{\sigma}-\dot{x}^{\sigma} d t$. Recall that $\varepsilon$ (or the system $\varepsilon_{\sigma}$ ) is said to be (locally) variational, if there exists a Lagrange function $\mathscr{L}=\mathscr{L}\left(t, q^{\sigma}, \dot{q}^{\sigma}\right)$ such that

$$
\varepsilon_{\sigma}=\frac{\partial \mathscr{L}}{\partial x^{\sigma}}-\frac{d}{d t} \frac{\partial \mathscr{L}}{\partial \dot{x}^{\sigma}}=\frac{\partial \mathscr{L}}{\partial x^{\sigma}}-\frac{\partial^{2} \mathscr{L}}{\partial t \partial \dot{x}^{\sigma}}-\frac{\partial^{2} \mathscr{L}}{\partial x^{\nu} \partial \dot{x}^{\sigma}} \dot{x}^{\nu}-\frac{\partial^{2} \mathscr{L}}{\partial \dot{x}^{\nu} \partial \dot{x}^{\sigma}} \ddot{x}^{\nu}
$$

Necessary and sufficient conditions for $\varepsilon$ to be variational are the well-known Helmholtz conditions, a system of partial differential equations for $\varepsilon_{\sigma}$,

$$
\begin{aligned}
& \frac{\partial \varepsilon_{\sigma}}{\partial \ddot{x}^{\nu}}-\frac{\partial \varepsilon_{\nu}}{\partial \ddot{x}^{\sigma}}=0, \quad \frac{\partial \varepsilon_{\sigma}}{\partial \dot{x}^{\nu}}+\frac{\partial \varepsilon_{\nu}}{\partial \dot{x}^{\sigma}}-\frac{d}{d t}\left(\frac{\partial \varepsilon_{\sigma}}{\partial \ddot{x}^{\nu}}+\frac{\partial \varepsilon_{\nu}}{\partial \ddot{x}^{\sigma}}\right)=0, \\
& \frac{\partial \varepsilon_{\sigma}}{\partial x^{\nu}}-\frac{\partial \varepsilon_{\nu}}{\partial x^{\sigma}}-\frac{1}{2} \frac{d}{d t}\left(\frac{\partial \varepsilon_{\sigma}}{\partial \dot{x}^{\nu}}-\frac{\partial \varepsilon_{\nu}}{\partial \dot{x}^{\sigma}}\right)=0 .
\end{aligned}
$$

Remark 4. The left-hand sides of equations (32), called the Helmholtz expressions, are the coefficients of the Helmholtz form in the variational sequence, see Sec. 3 . (24), (26).

Let $Q$ be an embedded submanifold of $\mathbb{R}^{m}, \operatorname{dim} Q=n, n \leq m, \iota: \mathbb{R} \times Q \rightarrow$ $\mathbb{R} \times \mathbb{R}^{m}$ be the canonical embedding, locally expressed by $t \circ \iota=t, x^{\sigma} \circ \iota=f^{\sigma}\left(q^{i}\right)$, $1 \leq i \leq n, 1 \leq \sigma \leq m$ (Sec. 2, (6) ). The second jet prolongation $J^{2} \iota$ (Sec. 2, (4)) has a chart expression given by Lemma 2. If $\varepsilon=\varepsilon_{\sigma} \omega^{\sigma} \wedge d t$ is a source form on $J^{2}\left(\mathbb{R} \times \mathbb{R}^{m}\right)$, we get a source form $J^{2} \iota^{*} \varepsilon$ on $J^{2}(\mathbb{R} \times Q)$,

$$
J^{2} \iota^{*} \varepsilon=\left(\varepsilon_{\sigma} \circ J^{2} \iota\right) J^{2} \iota^{*} \omega^{\sigma} \wedge J^{2} \iota^{*} d t=\tilde{\varepsilon}_{i} \eta^{i} \wedge d t,
$$

where $\eta^{i}=d q^{i}-\dot{q}^{i} d t$, and the coefficients $\tilde{\varepsilon}_{i}=\tilde{\varepsilon}_{i}\left(t, q^{j}, \dot{q}^{j}, \ddot{q}^{j}\right), 1 \leq i, j \leq n$, are given by

$$
\tilde{\varepsilon}_{i}=\left(\varepsilon_{\sigma} \circ J^{2} \iota\right) \frac{\partial f^{\sigma}}{\partial q^{i}} .
$$

Local variationality is characterized by means of the Helmholtz conditions: the induced source form $J^{2} \iota^{*} \varepsilon$ is locally variational if and only if the system of partial differential equations (32) is satisfied for functions $\tilde{\varepsilon}_{i}=\tilde{\varepsilon}_{i}\left(t, q^{j}, \dot{q}^{j}, \ddot{q}^{j}\right)$,

$$
\begin{aligned}
& \frac{\partial \tilde{\varepsilon}_{i}}{\partial \ddot{q}^{j}}-\frac{\partial \tilde{\varepsilon}_{j}}{\partial \ddot{q}^{i}}=0, \quad \frac{\partial \tilde{\varepsilon}_{i}}{\partial \dot{q}^{j}}+\frac{\partial \tilde{\varepsilon}_{j}}{\partial \dot{q}^{i}}-\frac{d}{d t}\left(\frac{\partial \tilde{\varepsilon}_{i}}{\partial \ddot{q}^{j}}+\frac{\partial \tilde{\varepsilon}_{j}}{\partial \ddot{q}^{i}}\right)=0, \\
& \frac{\partial \tilde{\varepsilon}_{i}}{\partial q^{j}}-\frac{\partial \tilde{\varepsilon}_{j}}{\partial q^{i}}-\frac{1}{2} \frac{d}{d t}\left(\frac{\partial \tilde{\varepsilon}_{i}}{\partial \dot{q}^{j}}-\frac{\partial \tilde{\varepsilon}_{j}}{\partial \dot{q}^{i}}\right)=0 .
\end{aligned}
$$

We have now the following two problems: (i) Suppose a submanifold $Q$ of $\mathbb{R}^{m}$ is fixed. Characterize a class of source forms $\varepsilon$ on $J^{2} \mathbb{R}^{m+1}$ such that $J^{2} \iota^{*} \varepsilon$ on $J^{2}(\mathbb{R} \times Q)$ is locally variational. (ii) Secondly, embedded submanifolds $Q$ of $\mathbb{R}^{m}$ may be regarded as unknowns of (35) for a given source form $\varepsilon$. In this case 
the Helmholtz conditions represent a system of partial differential equations for unknown functions $f^{\sigma}$.

We say that a submanifold $Q$ of $\mathbb{R}^{m}$ is variational for a source form $\varepsilon$, if the induced source form $J^{2} \iota^{*} \varepsilon$ is locally variational.

Necessary and sufficient conditions for variationality of the induced source form $J^{2} \iota^{*} \varepsilon$ give an implicit solution of both problems (i) and (ii). In the following theorem we consider a source form $\varepsilon$ (30) on $J^{2} \mathbb{R}^{m+1}$, defined by functions $\varepsilon_{\sigma}=$ $\varepsilon_{\sigma}\left(t, x^{\nu}, \dot{x}^{\nu}, \ddot{x}^{\nu}\right)$, and an embedded submanifold $Q$ of $\mathbb{R}^{m} \cdot(U, \varphi), \varphi=\left(t, q^{i}\right)$, is a fibered chart on $\mathbb{R} \times Q$, and $(V, \psi), \psi=\left(t, u^{j}, v^{\kappa}\right)$, is a chart on $\mathbb{R} \times \mathbb{R}^{m}$ adapted to $Q$.

Theorem 10 (Helmholtz conditions for induced variationality I). The following conditions are equivalent:

(a) The induced source form $J^{2} \iota^{*} \varepsilon$ is locally variational.

(b) If $\iota$ is expressed by the equations $t \circ \iota=t, x^{\sigma} \circ \iota=f^{\sigma}\left(q^{i}\right), 1 \leq \sigma \leq m$, then the functions $\varepsilon_{\sigma}$ and $f^{\sigma}$ satisfy

$$
\begin{aligned}
& \left(\frac{\partial \varepsilon_{\sigma}}{\partial \ddot{x}^{\nu}}-\frac{\partial \varepsilon_{\nu}}{\partial \ddot{x}^{\sigma}}\right)_{\circ J^{2} \iota}\left(\frac{\partial f^{\sigma}}{\partial q^{i}} \frac{\partial f^{\nu}}{\partial q^{j}}-\frac{\partial f^{\nu}}{\partial q^{i}} \frac{\partial f^{\sigma}}{\partial q^{j}}\right)=0, \\
& \left(\frac{\partial \varepsilon_{\sigma}}{\partial \dot{x}^{\nu}}+\frac{\partial \varepsilon_{\nu}}{\partial \dot{x}^{\sigma}}-\frac{d}{d t}\left(\frac{\partial \varepsilon_{\sigma}}{\partial \ddot{x}^{\nu}}+\frac{\partial \varepsilon_{\nu}}{\partial \ddot{x}^{\sigma}}\right)\right)_{\circ J^{2} \iota}\left(\frac{\partial f^{\sigma}}{\partial q^{i}} \frac{\partial f^{\nu}}{\partial q^{j}}+\frac{\partial f^{\nu}}{\partial q^{i}} \frac{\partial f^{\sigma}}{\partial q^{j}}\right) \\
& -\left(\frac{\partial \varepsilon_{\sigma}}{\partial \ddot{x}^{\nu}}-\frac{\partial \varepsilon_{\nu}}{\partial \ddot{x}^{\sigma}}\right)_{\circ J^{2} \iota}\left(\frac{\partial f^{\nu}}{\partial q^{j}} \frac{d}{d t} \frac{\partial f^{\sigma}}{\partial q^{i}}+\frac{\partial f^{\nu}}{\partial q^{i}} \frac{d}{d t} \frac{\partial f^{\sigma}}{\partial q^{j}}\right. \\
& \left.-\frac{\partial f^{\sigma}}{\partial q^{j}} \frac{d}{d t} \frac{\partial f^{\nu}}{\partial q^{i}}-\frac{\partial f^{\sigma}}{\partial q^{i}} \frac{d}{d t} \frac{\partial f^{\nu}}{\partial q^{j}}\right)=0,
\end{aligned}
$$

and

$$
\begin{aligned}
& \left(\frac{\partial \varepsilon_{\sigma}}{\partial x^{\nu}}-\frac{\partial \varepsilon_{\nu}}{\partial x^{\sigma}}-\frac{1}{2} \frac{d}{d t}\left(\frac{\partial \varepsilon_{\sigma}}{\partial \dot{x}^{\nu}}-\frac{\partial \varepsilon_{\nu}}{\partial \dot{x}^{\sigma}}\right)\right)_{\circ J^{2} \iota}\left(\frac{\partial f^{\sigma}}{\partial q^{i}} \frac{\partial f^{\nu}}{\partial q^{j}}-\frac{\partial f^{\nu}}{\partial q^{i}} \frac{\partial f^{\sigma}}{\partial q^{j}}\right) \\
& +\frac{1}{2}\left(\frac{\partial \varepsilon_{\sigma}}{\partial \dot{x}^{\nu}}+\frac{\partial \varepsilon_{\nu}}{\partial \dot{x}^{\sigma}}-\frac{d}{d t}\left(\frac{\partial \varepsilon_{\sigma}}{\partial \ddot{x}^{\nu}}+\frac{\partial \varepsilon_{\nu}}{\partial \ddot{x}^{\sigma}}\right)\right)_{\circ J^{2} \iota} \\
& \cdot\left(\frac{\partial f^{\sigma}}{\partial q^{i}} \frac{d}{d t} \frac{\partial f^{\nu}}{\partial q^{j}}-\frac{\partial f^{\sigma}}{\partial q^{j}} \frac{d}{d t} \frac{\partial f^{\nu}}{\partial q^{i}}+\frac{\partial f^{\nu}}{\partial q^{i}} \frac{d}{d t} \frac{\partial f^{\sigma}}{\partial q^{j}}-\frac{\partial f^{\nu}}{\partial q^{j}} \frac{d}{d t} \frac{\partial f^{\sigma}}{\partial q^{i}}\right) \\
& -\frac{1}{2} \frac{d}{d t}\left(\frac{\partial \varepsilon_{\sigma}}{\partial \ddot{x}^{\nu}}-\frac{\partial \varepsilon_{\nu}}{\partial \ddot{x}^{\sigma}}\right)_{\circ J^{2} \iota} \frac{d}{d t}\left(\frac{\partial f^{\sigma}}{\partial q^{i}} \frac{\partial f^{\nu}}{\partial q^{j}}-\frac{\partial f^{\nu}}{\partial q^{i}} \frac{\partial f^{\sigma}}{\partial q^{j}}\right) \\
& +\left(\frac{\partial \varepsilon_{\sigma}}{\partial \ddot{x}^{\nu}}-\frac{\partial \varepsilon_{\nu}}{\partial \ddot{x}^{\sigma}}\right)_{\circ J^{2} \iota}\left(\frac{d}{d t}\left(\frac{\partial f^{\sigma}}{\partial q^{j}}\right) \frac{d}{d t}\left(\frac{\partial f^{\nu}}{\partial q^{i}}\right)\right. \\
& \left.-\frac{d}{d t}\left(\frac{\partial f^{\nu}}{\partial q^{j}}\right) \frac{d}{d t}\left(\frac{\partial f^{\sigma}}{\partial q^{i}}\right)\right)=0 .
\end{aligned}
$$

(c) If $\iota$ is expressed by the equations $t \circ \iota=t, u^{j} \circ \iota=\left.u^{j}\right|_{V \cap(\mathbb{R} \times Q)}, v^{\kappa} \circ \iota=c^{\kappa}$, where $1 \leq j \leq n, n+1 \leq \kappa \leq m$, and $c^{\kappa}$ are some real numbers, then the functions $\varepsilon_{\sigma}$ satisfy

$$
\begin{aligned}
& \left(\frac{\partial \varepsilon_{i}}{\partial \ddot{u}^{j}}-\frac{\partial \varepsilon_{j}}{\partial \ddot{u}^{i}}\right)_{\circ J^{2} \iota}=0, \quad\left(\frac{\partial \varepsilon_{i}}{\partial \dot{u}^{j}}+\frac{\partial \varepsilon_{j}}{\partial \dot{u}^{i}}-\frac{d}{d t}\left(\frac{\partial \varepsilon_{i}}{\partial \ddot{u}^{j}}+\frac{\partial \varepsilon_{j}}{\partial \ddot{u}^{i}}\right)\right)_{\circ J^{2} \iota}=0, \\
& \left(\frac{\partial \varepsilon_{i}}{\partial u^{j}}-\frac{\partial \varepsilon_{j}}{\partial u^{i}}-\frac{1}{2} \frac{d}{d t}\left(\frac{\partial \varepsilon_{i}}{\partial \dot{u}^{j}}-\frac{\partial \varepsilon_{j}}{\partial \dot{u}^{i}}\right)\right)_{\circ J^{2} \iota}=0 .
\end{aligned}
$$


Proof. Equivalence of both conditions (b) and (c) with (a) follows from a straightforward substitution of the expressions for $\tilde{\varepsilon}_{i}$ (34) into (35) with respect to the considered charts.

Remark 5. Note that the Helmholtz expressions (32) and (35) appear in Theorem 10. condition (b) contains linear combinations of expressions (32), and in (c), (36) contains expressions (35), restricted to a submanifold. In particular, the equivalence conditions of Theorem 10 show that local variationality of the induced system $\tilde{\varepsilon}_{i}$ (34) determines the structure of the initial system on $J^{2} \mathbb{R}^{m+1}$, defined by functions $\varepsilon_{\sigma}=\varepsilon_{\sigma}\left(t, x^{\nu}, \dot{x}^{\nu}, \ddot{x}^{\nu}\right)$, and vice-versa. Namely, the system $\varepsilon_{\sigma}, \sigma=1,2, \ldots, m$, must contain a subsystem of $n$ equations for $n$ dependent variables, which is locally variational when contracted to a submanifold. This observation gives a straightforward method to construct systems of differential equations on Euclidean spaces, which are not variational but induce locally variational systems on submanifolds.

Corollary 2. Suppose a source form $\varepsilon=\varepsilon_{\sigma} \omega^{\sigma} \wedge d t$ (30) on $J^{2} \mathbb{R}^{m+1}$ is variational. Then the induced source form $J^{2} \iota^{*} \varepsilon=\tilde{\varepsilon}_{i} \eta^{i} \wedge d t$ (33) on $J^{2}(\mathbb{R} \times Q)$ is globally variational. Moreover, if $\mathscr{L}=\mathscr{L}\left(t, x^{\nu}, \dot{x}^{\nu}\right)$ is a Lagrange function of the system $\varepsilon_{\sigma}$, then $\mathscr{L} \circ J^{1} \iota$ is a Lagrange function of the induced system $\tilde{\varepsilon}_{i}$.

Proof. From Theorem [10 it follows that $\tilde{\varepsilon}_{i}$ satisfies the Helmholtz conditions (35) provided $\varepsilon_{\sigma}$ is satisfies (32). Suppose now that for some function $\mathscr{L}: J^{1} \mathbb{R}^{m+1} \rightarrow \mathbb{R}$, $\varepsilon_{\sigma}$ are expressed as the Euler-Lagrange expressions (31). We show that the EulerLagrange expressions of the function $\mathscr{L} \circ J^{1} \iota: J^{1}(\mathbb{R} \times Q) \rightarrow \mathbb{R}$ coincide with $\tilde{\varepsilon}_{i}$. Using the chart expression of $J^{1} \iota$ we get

$$
\begin{aligned}
& \frac{\partial\left(\mathscr{L} \circ J^{1} \iota\right)}{\partial q^{i}}-\frac{d}{d t} \frac{\partial\left(\mathscr{L} \circ J^{1} \iota\right)}{\partial \dot{q}^{i}} \\
& =\left(\frac{\partial \mathscr{L}}{\partial x^{\sigma}}\right)_{\circ J^{1} \iota} \frac{\partial f^{\sigma}}{\partial q^{i}}+\left(\frac{\partial \mathscr{L}}{\partial \dot{x}^{\sigma}}\right)_{\circ J^{1} \iota} \frac{\partial^{2} f^{\sigma}}{\partial q^{i} \partial q^{j}} \dot{q}^{j}-\frac{d}{d t}\left(\frac{\partial \mathscr{L}}{\partial \dot{x}^{\sigma}}{ }_{\circ J^{1} \iota} \frac{\partial f^{\sigma}}{\partial q^{i}}\right) \\
& =\left(\frac{\partial \mathscr{L}}{\partial x^{\sigma}}-\frac{d}{d t} \frac{\partial \mathscr{L}}{\partial \dot{x}^{\sigma}}\right)_{\circ J^{2} \iota} \frac{\partial f^{\sigma}}{\partial q^{i}}=\left(\varepsilon_{\sigma} \circ J^{2} \iota\right) \frac{\partial f^{\sigma}}{\partial q^{i}}=\tilde{\varepsilon}_{i},
\end{aligned}
$$

as required.

It is easily seen that variationality of a source form $\varepsilon$ (30) implies that its coefficients $\varepsilon_{\sigma}$ are linear in the second derivatives, i.e.

$$
\varepsilon_{\sigma}=A_{\sigma}+B_{\sigma \nu} \ddot{x}^{\nu}
$$

for some functions $A_{\sigma}, B_{\sigma \nu}$ depending $t, x^{\sigma}$ and $\dot{x}^{\sigma}$ only. In this case the Helmholtz conditions (32) are equivalent with the following conditions for $A_{\sigma}$ and $B_{\sigma \nu}$ (cf. Sarlet [10]),

$$
\begin{aligned}
& B_{\sigma \nu}=B_{\nu \sigma}, \quad \frac{\partial B_{\sigma \kappa}}{\partial \dot{x}^{\nu}}=\frac{\partial B_{\nu \kappa}}{\partial \dot{x}^{\sigma}}, \\
& \frac{\partial A_{\sigma}}{\partial \dot{x}^{\nu}}+\frac{\partial A_{\nu}}{\partial \dot{x}^{\sigma}}-2\left(\frac{\partial B_{\sigma \nu}}{\partial t}+\frac{\partial B_{\sigma \nu}}{\partial x^{\kappa}} \dot{x}^{\kappa}\right)=0, \\
& \frac{\partial A_{\sigma}}{\partial x^{\nu}}-\frac{\partial A_{\nu}}{\partial x^{\sigma}}-\frac{1}{2}\left(\frac{\partial}{\partial t}\left(\frac{\partial A_{\sigma}}{\partial \dot{x}^{\nu}}-\frac{\partial A_{\nu}}{\partial \dot{x}^{\sigma}}\right)+\frac{\partial}{\partial x^{\kappa}}\left(\frac{\partial A_{\sigma}}{\partial \dot{x}^{\nu}}-\frac{\partial A_{\nu}}{\partial \dot{x}^{\sigma}}\right) \dot{x}^{\kappa}\right)=0 .
\end{aligned}
$$

From now on we shall consider second-order systems (37) with coefficients $A_{\sigma}$ and $B_{\sigma \nu}$ of the variables $x^{\sigma}$ and $\dot{x}^{\sigma}$, and not depending on time variable $t$ explicitly. It 
is straightforward to check that the coefficients of the induced source form $J^{2} \iota^{*} \varepsilon=$ $\tilde{\varepsilon}_{j} \eta^{j} \wedge d t(33)$ are expressed by $\tilde{\varepsilon}_{j}=P_{j}+Q_{j k} \ddot{q}^{k}$, where

$$
P_{j}=\tilde{A}_{\sigma} \frac{\partial f^{\sigma}}{\partial q^{j}}+\tilde{B}_{\sigma \nu} \frac{\partial f^{\sigma}}{\partial q^{j}} \frac{\partial^{2} f^{\nu}}{\partial q^{i} \partial q^{k}} \dot{q}^{i} \dot{q}^{k}, \quad Q_{j k}=\tilde{B}_{\sigma \nu} \frac{\partial f^{\sigma}}{\partial q^{j}} \frac{\partial f^{\nu}}{\partial q^{k}},
$$

and $\tilde{A}_{\sigma}=A_{\sigma} \circ J^{1} \iota, \tilde{B}_{\sigma \nu}=B_{\sigma \nu} \circ J^{1} \iota$. Suppose $\varepsilon$ is a source form on $J^{2} \mathbb{R}^{m+1}$, defined by functions (37), $(U, \varphi), \varphi=\left(t, q^{i}\right)$, is a fibered chart on $\mathbb{R} \times Q$, and $(V, \psi), \psi=\left(t, u^{j}, v^{\kappa}\right)$, is a chart on $\mathbb{R} \times \mathbb{R}^{m}$ adapted to $Q$.

Theorem 11 (Helmholtz conditions for induced variationality II). The following conditions are equivalent:

(a) The induced source form $J^{2} \iota^{*} \varepsilon$ is locally variational.

(b) If $\iota$ is expressed by the equations $t \circ \iota=t, x^{\sigma} \circ \iota=f^{\sigma}\left(q^{i}\right), 1 \leq \sigma \leq m$, then the functions $\tilde{A}_{\sigma}, \tilde{B}_{\sigma \nu}$, and $f^{\sigma}$ satisfy

$$
\begin{aligned}
& \left(\tilde{B}_{\sigma \nu}-\tilde{B}_{\nu \sigma}\right) \frac{\partial f^{\sigma}}{\partial q^{j}} \frac{\partial f^{\nu}}{\partial q^{k}}=0, \quad\left(\frac{\partial \tilde{B}_{\sigma \nu}}{\partial \dot{q}^{i}} \frac{\partial f^{\sigma}}{\partial q^{j}}-\frac{\partial \tilde{B}_{\sigma \nu}}{\partial \dot{q}^{j}} \frac{\partial f^{\sigma}}{\partial q^{i}}\right) \frac{\partial f^{\nu}}{\partial q^{k}}=0, \\
& \frac{\partial \tilde{A}_{\sigma}}{\partial \dot{q}^{j}} \frac{\partial f^{\sigma}}{\partial q^{i}}+\frac{\partial \tilde{A}_{\sigma}}{\partial \dot{q}^{i}} \frac{\partial f^{\sigma}}{\partial q^{j}}-2 \frac{\partial \tilde{B}_{\sigma \nu}}{\partial q^{k}} \frac{\partial f^{\sigma}}{\partial q^{i}} \frac{\partial f^{\nu}}{\partial q^{j}} \dot{q}^{k} \\
& +\left(\frac{\partial \tilde{B}_{\sigma \nu}}{\partial \dot{q}^{j}} \frac{\partial f^{\sigma}}{\partial q^{i}}+\frac{\partial \tilde{B}_{\sigma \nu}}{\partial \dot{q}^{i}} \frac{\partial f^{\sigma}}{\partial q^{j}}\right) \frac{\partial^{2} f^{\nu}}{\partial q^{k} \partial q^{l}} \dot{q}^{k} \dot{q}^{l}=0,
\end{aligned}
$$

and

$$
\begin{aligned}
& \frac{\partial \tilde{A}_{\sigma}}{\partial q^{j}} \frac{\partial f^{\sigma}}{\partial q^{i}}-\frac{\partial \tilde{A}_{\sigma}}{\partial q^{i}} \frac{\partial f^{\sigma}}{\partial q^{j}}-\frac{1}{2} \frac{\partial}{\partial q^{k}}\left(\frac{\partial \tilde{A}_{\sigma}}{\partial \dot{q}^{j}} \frac{\partial f^{\sigma}}{\partial q^{i}}-\frac{\partial \tilde{A}_{\sigma}}{\partial \dot{q}^{i}} \frac{\partial f^{\sigma}}{\partial q^{j}}\right) \dot{q}^{k} \\
& -\left(\tilde{B}_{\sigma \nu}-\tilde{B}_{\nu \sigma}\right) \frac{\partial^{2} f^{\sigma}}{\partial q^{i} \partial q^{l}} \frac{\partial^{2} f^{\nu}}{\partial q^{j} \partial q^{k}} \dot{q}^{k} \dot{q}^{l} \\
& -\frac{\partial \tilde{B}_{\sigma \nu}}{\partial q^{k}}\left(\frac{\partial f^{\sigma}}{\partial q^{i}} \frac{\partial^{2} f^{\nu}}{\partial q^{l} \partial q^{j}}-\frac{\partial f^{\sigma}}{\partial q^{j}} \frac{\partial^{2} f^{\nu}}{\partial q^{l} \partial q^{i}}\right) \dot{q}^{k} \dot{q}^{l} \\
& +\left(\frac{\partial \tilde{B}_{\sigma \nu}}{\partial q^{j}} \frac{\partial f^{\sigma}}{\partial q^{i}}-\frac{\partial \tilde{B}_{\sigma \nu}}{\partial q^{i}} \frac{\partial f^{\sigma}}{\partial q^{j}}\right) \frac{\partial^{2} f^{\nu}}{\partial q^{k} \partial q^{l}} \dot{q}^{k} \dot{q}^{l} \\
& -\frac{1}{2} \frac{\partial}{\partial q^{k}}\left(\left(\frac{\partial \tilde{B}_{\sigma \nu}}{\partial \dot{q}^{j}} \frac{\partial f^{\sigma}}{\partial q^{i}}-\frac{\partial \tilde{B}_{\sigma \nu}}{\partial \dot{q}^{i}} \frac{\partial f^{\sigma}}{\partial q^{j}}\right) \frac{\partial^{2} f^{\nu}}{\partial q^{s} \partial q^{l}} \dot{q}^{s} \dot{q}^{l}\right) \dot{q}^{k}=0 .
\end{aligned}
$$

(c) If $\iota$ is expressed by the equations $t \circ \iota=t, u^{j} \circ \iota=\left.u^{j}\right|_{V \cap(\mathbb{R} \times Q)}, v^{\kappa} \circ \iota=c^{\kappa}$, where $1 \leq j \leq n, n+1 \leq \kappa \leq m$, and $c^{\kappa}$ are some real numbers, then $\tilde{A}_{j}=A_{j} \circ J^{1} \iota$, $\tilde{B}_{j k}=B_{j k} \circ J^{1} \iota, 1 \leq j, k \leq n$, satisfy

$$
\begin{aligned}
& \tilde{B}_{j k}=\tilde{B}_{k j}, \quad \frac{\partial \tilde{B}_{j l}}{\partial \dot{q}^{k}}=\frac{\partial \tilde{B}_{k l}}{\partial \dot{q}^{j}}, \quad \frac{\partial \tilde{A}_{j}}{\partial \dot{q}^{k}}+\frac{\partial \tilde{A}_{k}}{\partial \dot{q}^{j}}-2 \frac{\partial \tilde{B}_{j k}}{\partial q^{l}} \dot{q}^{l}=0, \\
& \frac{\partial \tilde{A}_{j}}{\partial q^{k}}-\frac{\partial \tilde{A}_{k}}{\partial q^{j}}-\frac{1}{2} \frac{\partial}{\partial q^{l}}\left(\frac{\partial \tilde{A}_{j}}{\partial \dot{q}^{k}}-\frac{\partial \tilde{A}_{k}}{\partial \dot{q}^{j}}\right) \dot{q}^{l}=0 .
\end{aligned}
$$

Proof. The proof follows from a direct application of the Helmholtz variationality conditions (38), applied to coefficients (39) of $J^{2} \iota^{*} \varepsilon$. 
Corollary 3. Let $Q$ be a one-dimensional embedded submanifold of $\mathbb{R}^{m}$. Then the induced source form $J^{2} \iota^{*} \varepsilon$ on an open subset of $J^{2}(\mathbb{R} \times Q)$ is locally variational if and only if the functions $A_{\sigma}, B_{\sigma \nu}$, and $f^{\sigma}$ satisfy

$$
\begin{aligned}
& \left(\frac{\partial A_{\sigma}}{\partial \dot{x}^{\nu}}\right)_{\circ J^{1} \iota} \frac{\partial f^{\nu}}{\partial q} \frac{\partial f^{\sigma}}{\partial q}+\left(\frac{\partial B_{\sigma \kappa}}{\partial \dot{x}^{\nu}}-\frac{\partial B_{\sigma \nu}}{\partial \dot{x}^{\kappa}}\right)_{\circ J^{1} \iota} \dot{q} \frac{\partial f^{\nu}}{\partial q} \frac{\partial f^{\sigma}}{\partial q} \frac{d}{d t} \frac{\partial f^{\kappa}}{\partial q} \\
& +\left(B_{\sigma \nu}-B_{\nu \sigma}\right)_{\circ J^{1} \iota} \frac{\partial f^{\sigma}}{\partial q} \frac{d}{d t} \frac{\partial f^{\nu}}{\partial q}-\left(\frac{\partial B_{\sigma \nu}}{\partial x^{\kappa}}\right)_{\circ J^{1} \iota} \frac{d f^{\kappa}}{d t} \frac{\partial f^{\nu}}{\partial q} \frac{\partial f^{\sigma}}{\partial q}=0 .
\end{aligned}
$$

Remark 6 . We point out that the equivalent conditions of Theorem 11 restrict not only the structure of the initial source form $\varepsilon$, but also a submanifold $Q$ of $\mathbb{R}^{m}$ to be found. In particular, we observe that (41) is satisfied identically for systems $\varepsilon_{\sigma}$ such that (i) $A_{\sigma}=A_{\sigma}\left(x^{\kappa}\right)$, (ii) $B_{\sigma \nu}=B_{\sigma \nu}\left(\dot{x}^{\kappa}\right)$, and

$$
B_{\sigma \nu}=B_{\nu \sigma}, \quad \frac{\partial B_{\sigma \nu}}{\partial \dot{x}^{\kappa}}=\frac{\partial B_{\sigma \kappa}}{\partial \dot{x}^{\nu}} .
$$

In other words, second-order systems of ordinary equations linear in second derivatives which satisfy conditions (i), (ii), and (42) are variational when contracted to arbitrary one-dimensional submanifold of the Euclidean space $\mathbb{R}^{m}$. Conditions (42) belong to the set of the Helmholtz conditions (38) for $\varepsilon_{\sigma}$, nevertheless (42) together with (i) and (ii) do not imply (local) variationality of the system $\varepsilon_{\sigma}$ (37).

Remark 7 (Forces). Important particular case of (37) are systems solved with respect to the second derivatives,

$$
\ddot{x}^{\sigma}=F^{\sigma}
$$

where $1 \leq \sigma, \nu \leq m$, and $F^{\sigma}=F^{\sigma}\left(x^{\nu}, \dot{x}^{\nu}\right)$ are given functions (called mechanical forces). To rewrite (43) into the form $\varepsilon_{\sigma}=0$, we put $B_{\sigma \nu}=\delta_{\sigma \nu}$ (the Kronecker symbol), and $A_{\sigma}=-\delta_{\sigma \nu} F^{\nu}$. From Corollary 3 we obtain a necessary and sufficient condition for a one-dimensional submanifold $Q$ of $\mathbb{R}^{m}$ to be a variational submanifold for (43),

$$
\sum_{\sigma}\left(\frac{\partial F^{\sigma}}{\partial \dot{x}^{\nu}}\right)_{\circ J^{1} \iota} \frac{\partial f^{\sigma}}{\partial q} \frac{\partial f^{\nu}}{\partial q}=0
$$

In particular, if the forces $F^{\sigma}$ do not depend on velocities $\dot{x}^{\nu}$, then clearly any one-dimensional submanifold is variational for (43).

\section{VARIATIONAL SUBMANIFOLDS: EXAMPLES}

We illustrate the general theory on simple examples of holonomic constraints in mechanics. Consider the Euclidean space $\mathbb{R} \times \mathbb{R}^{3}$ endowed with the canonical coordinates $(t, x, y, z)$, and the associated fibered coordinates on the jet prolongations $J^{1}\left(\mathbb{R} \times \mathbb{R}^{3}\right), J^{2}\left(\mathbb{R} \times \mathbb{R}^{3}\right)$, denoted by $(t, x, y, z, \dot{x}, \dot{y}, \dot{z})$ and $(t, x, y, z, \dot{x}, \dot{y}, \dot{z}, \ddot{x}, \ddot{y}, \ddot{z})$, respectively.

In Examples 1 and 2 we consider two topologically non-equivalent, embedded submanifolds of $\mathbb{R}^{3}$, the sphere $S^{2}$ and the Möbius strip $M_{r, a}$. With the help of the variational sequence theory (Sec. 3) the concepts of local and global variationality of the constrained systems of equations are studied. Then in Examples 3-8 we study one- and two-dimensional variational submanifolds of Euclidean spaces for concrete systems of second-order differential equations. 
Example 1 (Induced variational equations on 2-sphere). Denote by $S_{r_{0}}^{2}$ (resp. $S^{2}$ ) the two-dimensional sphere in $\mathbb{R}^{3}$ of radius $r_{0}>0$ (resp. unit sphere) with centre in the origin. In the standard sense, the open submanifold $\mathbb{R} \times\left(\mathbb{R}^{3} \backslash\{(0,0,0)\}\right)$ of $\mathbb{R}^{4}$ is endowed with a smooth atlas, which consists of two spherical charts adapted to the embedded submanifold $\mathbb{R} \times S_{r_{0}}^{2}$ for every $r_{0}$. The equations $t=t, x=$ $r \cos \varphi \sin \vartheta, y=r \sin \varphi \sin \vartheta, z=r \cos \vartheta$, define a diffeomorphism $U \ni(t, r, \varphi, \vartheta) \rightarrow$ $(t, x(r, \varphi, \vartheta), y(r, \varphi, \vartheta), z(r, \varphi, \vartheta)) \in V$, where for instance $U=\left\{(t, r, \varphi, \vartheta) \in \mathbb{R}^{3} \mid t \in\right.$ $\mathbb{R}, r>0,0<\varphi<2 \pi, 0<\vartheta<\pi\}$ maps onto an open subset $V=\mathbb{R} \times \mathbb{R}^{3} \backslash$ $\left\{(x, y, z) \in \mathbb{R}^{3} \mid x \geq 0, y=0\right\}$ of $\mathbb{R}^{4}$. The inverse transformation $V \ni(t, x, y, z) \rightarrow$ $(t, r, \varphi, \vartheta) \in U$, is a diffeomorphism expressed as

$$
\begin{aligned}
& t=t, \quad r=\sqrt{x^{2}+y^{2}+z^{2}}, \quad \vartheta=\arccos \frac{z}{\sqrt{x^{2}+y^{2}+z^{2}}} \\
& \varphi= \begin{cases}\arccos \frac{x}{\sqrt{x^{2}+y^{2}}}, & y>0, \\
2 \pi-\arccos \frac{x}{\sqrt{x^{2}+y^{2}}}, & y<0, \\
\pi, & y=0, x<0 .\end{cases}
\end{aligned}
$$

$(V, \Psi), \Psi=(t, r, \varphi, \vartheta)$, is a chart on $\mathbb{R} \times\left(\mathbb{R}^{3} \backslash\{(0,0,0)\}\right.$, adapted to $\mathbb{R} \times S_{r_{0}}^{2}$. We complete $(V, \Psi)$ by a rotated chart $(\bar{V}, \bar{\Psi})$, where $\bar{V}=\mathbb{R} \times\left(\mathbb{R}^{3} \backslash\left\{(x, y, z) \in \mathbb{R}^{3} \mid x \leq\right.\right.$ $0, z=0\}), \bar{\Psi}=(\bar{t}, \bar{r}, \bar{\varphi}, \bar{\vartheta})$, in order to obtain an atlas on $\mathbb{R} \times\left(\mathbb{R}^{3} \backslash\{(0,0,0)\}\right.$. The coordinate transformation $\Psi \circ \bar{\Psi}^{-1}: \bar{\Psi}(V \cap \bar{V}) \rightarrow \Psi(V \cap \bar{V})$, is expressed by

$$
t=\bar{t}, \quad r=\bar{r}, \quad \cos \varphi=\frac{-\cos \bar{\varphi} \sin \bar{\vartheta}}{\sqrt{1-\sin ^{2} \bar{\varphi} \sin ^{2} \bar{\vartheta}}}, \quad \cos \vartheta=-\sin \bar{\varphi} \sin \bar{\vartheta} .
$$

Consider the canonical embedding $\iota: \mathbb{R} \times S^{2} \rightarrow \mathbb{R} \times \mathbb{R}^{3}$ over the identity of the real line $\mathbb{R}$. Let $\varepsilon$ be a source form on $J^{2}\left(\mathbb{R} \times \mathbb{R}^{3}\right), \varepsilon=\varepsilon_{x} \omega^{x} \wedge d t+\varepsilon_{y} \omega^{y} \wedge d t+\varepsilon_{z} \omega^{z} \wedge d t$, where $\omega^{x}=d x-\dot{x} d t, \omega^{y}=d y-\dot{y} d t, \omega^{z}=d z-\dot{z} d t$. We discuss variationality of $J^{2} \iota^{*} \varepsilon$.

(i) If $\varepsilon$ is locally variational, then by Corollary 1 it is also globally variational since $H_{\mathrm{deR}}^{2}\left(\mathbb{R} \times \mathbb{R}^{3}\right)$ is trivial. Thus, $\varepsilon$ coincides with the Euler-Lagrange form $E_{\lambda}$, associated with a Lagrangian $\lambda=\mathscr{L} d t$, where $\mathscr{L}: J^{1}\left(\mathbb{R} \times \mathbb{R}^{3}\right) \rightarrow \mathbb{R}$ is a globally defined function. By Corollary 2 the induced source form $J^{2} \iota^{*} \varepsilon$ is globally variational and coincides with the Euler-Lagrange form, associated with the Lagrangian $J^{2} \iota^{*} \lambda$. From Theorem 9] (a), we get an expression of the constrained Euler-Lagrange form with respect to $\left(V^{1}, \Psi^{1}\right)$,

$$
\begin{aligned}
& J^{2} \iota^{*} \varepsilon=J^{2} \iota^{*} E_{\lambda} \\
& =\left(-\left(E_{x}(\mathscr{L}) \circ J^{2} \iota\right) \sin \varphi \sin \vartheta+\left(E_{y}(\mathscr{L}) \circ J^{2} \iota\right) \cos \varphi \sin \vartheta\right) \eta^{\varphi} \wedge d t \\
& +\left(\left(E_{x}(\mathscr{L}) \circ J^{2} \iota\right) \cos \varphi \cos \vartheta+\left(E_{y}(\mathscr{L}) \circ J^{2} \iota\right) \sin \varphi \cos \vartheta\right. \\
& \left.-\left(E_{z}(\mathscr{L}) \circ J^{2} \iota\right) \sin \vartheta\right) \eta^{\vartheta} \wedge d t,
\end{aligned}
$$

where $\eta^{\varphi}=d \varphi-\dot{\varphi} d t, \eta^{\vartheta}=d \vartheta-\dot{\vartheta} d t$, and

$$
\begin{aligned}
& E_{x}(\mathscr{L})=\frac{\partial \mathscr{L}}{\partial x}-\frac{d}{d t} \frac{\partial \mathscr{L}}{\partial \dot{x}}, \quad E_{y}(\mathscr{L})=\frac{\partial \mathscr{L}}{\partial y}-\frac{d}{d t} \frac{\partial \mathscr{L}}{\partial \dot{y}}, \\
& E_{z}(\mathscr{L})=\frac{\partial \mathscr{L}}{\partial z}-\frac{d}{d t} \frac{\partial \mathscr{L}}{\partial \dot{z}} .
\end{aligned}
$$


(ii) Suppose $\varepsilon$ is not variational. If $\varepsilon=\varepsilon_{\varphi} \omega^{\varphi} \wedge d t+\varepsilon_{\vartheta} \omega^{\vartheta} \wedge d t+\varepsilon_{r} \omega^{r} \wedge d t$ in $\left(V^{1}, \Psi^{1}\right)$, we get necessary and sufficient conditions (Theorem 10) for local variationality of $J^{2} \iota^{*} \varepsilon$ as

$$
\begin{aligned}
& \left(\frac{\partial \varepsilon_{\varphi}}{\partial \ddot{\vartheta}}-\frac{\partial \varepsilon_{\vartheta}}{\partial \ddot{\varphi}}\right)_{\circ J^{2} \iota}=0, \\
& \left(\frac{\partial \varepsilon_{\varphi}}{\partial \dot{\varphi}}-\frac{d}{d t} \frac{\partial \varepsilon_{\varphi}}{\partial \ddot{\varphi}}\right)_{\circ J^{2} \iota}=0, \quad\left(\frac{\partial \varepsilon_{\vartheta}}{\partial \dot{\vartheta}}-\frac{d}{d t} \frac{\partial \varepsilon_{\vartheta}}{\partial \ddot{\vartheta}}\right)_{\circ J^{2} \iota}=0, \\
& \left(\frac{\partial \varepsilon_{\varphi}}{\partial \dot{\vartheta}}+\frac{\partial \varepsilon_{\vartheta}}{\partial \dot{\varphi}}-\frac{d}{d t}\left(\frac{\partial \varepsilon_{\varphi}}{\partial \ddot{\vartheta}}+\frac{\partial \varepsilon_{\vartheta}}{\partial \ddot{\varphi}}\right)\right)_{\circ J^{2} \iota}=0, \\
& \left(\frac{\partial \varepsilon_{\varphi}}{\partial \vartheta}-\frac{\partial \varepsilon_{\vartheta}}{\partial \varphi}-\frac{1}{2} \frac{d}{d t}\left(\frac{\partial \varepsilon_{\varphi}}{\partial \dot{\vartheta}}-\frac{\partial \varepsilon_{\vartheta}}{\partial \dot{\varphi}}\right)\right)_{\circ J^{2} \iota}=0 .
\end{aligned}
$$

Since the second de Rham cohomology group of $S^{2}$ is non-trivial, $H_{\mathrm{deR}}^{2}\left(\mathbb{R} \times S^{2}\right)=\mathbb{R}$, we observe by Corollary 1 that the local variationality conditions (44) do not assure global variationality of $J^{2} \iota^{*} \varepsilon$. Apparently, one can easily find other examples of embedded submanifolds in Euclidean spaces possessing topological obstructions for global variationality.

Example 2 (Induced variational equations on Möbius strip). Let $M_{r, a}$ denotes the Möbius strip in $\mathbb{R}^{3}$ (without boundary) of radius $r$ and wideness $2 a$, where $0<a<$ $r$. We introduce a smooth atlas on open subset $\mathbb{R} \times\left(\mathbb{R}^{3} \backslash\{(0,0, z) \mid z \in \mathbb{R}\}\right)$ of $\mathbb{R}^{4}$, consisting of adapted charts to embedded submanifold $\mathbb{R} \times M_{r, a}$ as follows. The equations $t=t$, and

$$
\begin{aligned}
& x=r \cos \varphi+\tau \cos \frac{\varphi}{2} \cos \varphi-\kappa \sin \frac{\varphi}{2} \cos \varphi, \\
& y=r \sin \varphi+\tau \cos \frac{\varphi}{2} \sin \varphi-\kappa \sin \frac{\varphi}{2} \sin \varphi, \\
& z=\tau \sin \frac{\varphi}{2}+\kappa \cos \frac{\varphi}{2}, \quad 0<\varphi<2 \pi, \quad-\infty<\tau<+\infty,
\end{aligned}
$$

where

$$
-\infty<\kappa<\frac{\cos (\varphi / 2)}{\sin (\varphi / 2)} \tau+\frac{r}{\sin (\varphi / 2)}
$$

define a diffeomorphism

$$
U \ni(t, \varphi, \tau, \kappa) \rightarrow(t, x(\varphi, \tau, \kappa), y(\varphi, \tau, \kappa), z(\varphi, \tau, \kappa)) \in V,
$$

where $V=\mathbb{R} \times\left(\mathbb{R}^{3} \backslash\{[0,+\infty) \times\{0\} \times \mathbb{R}\}\right), U=\mathbb{R} \times U_{0}$, and $U_{0}$ is an open subset of $\mathbb{R}^{3}$, given by condition (45). We get a chart $(V, \Psi), \Psi=(t, \varphi, \tau, \kappa)$, on $\mathbb{R} \times\left(\mathbb{R}^{3} \backslash\{(0,0, z) \mid z \in \mathbb{R}\}\right)$, adapted to submanifold $\mathbb{R} \times M_{r, a}$, which is characterized by $\kappa=0$. Another adapted chart to $\mathbb{R} \times M_{r, a}$, is given by $(\bar{V}, \bar{\Psi}), \bar{\Psi}=(t, \bar{\varphi}, \bar{\tau}, \bar{\kappa})$, where $\bar{V}=\mathbb{R} \times\left(\mathbb{R}^{3} \backslash\{(-\infty, 0] \times\{0\} \times \mathbb{R}\}\right)$, and

$$
\begin{aligned}
& x=r \cos \bar{\varphi}+\bar{\tau} \cos \frac{\bar{\varphi}}{2} \cos \bar{\varphi}-\bar{\kappa} \sin \frac{\bar{\varphi}}{2} \cos \bar{\varphi}, \\
& y=r \sin \bar{\varphi}+\bar{\tau} \cos \frac{\bar{\varphi}}{2} \sin \bar{\varphi}-\bar{\kappa} \sin \frac{\bar{\varphi}}{2} \sin \bar{\varphi}, \\
& z=\bar{\tau} \sin \frac{\bar{\varphi}}{2}+\bar{\kappa} \cos \frac{\bar{\varphi}}{2}, \quad-\pi<\bar{\varphi}<\pi,-\infty<\kappa<+\infty,
\end{aligned}
$$

where

$$
\frac{\sin (\varphi / 2)}{\cos (\varphi / 2)} \kappa-\frac{r}{\cos (\varphi / 2)}<\tau<+\infty
$$


Then $(V, \Psi)$ and $(\bar{V}, \bar{\Psi})$ define an adapted atlas to $\mathbb{R} \times M_{r, a}$, obeying chart transformation

$$
\bar{\Psi} \circ \Psi^{-1}(t, \varphi, \tau, \kappa)= \begin{cases}(t, \varphi, \tau, \kappa), & \varphi \in(0, \pi), \\ (t, \varphi-2 \pi,-\tau,-\kappa), & \varphi \in(\pi, 2 \pi) .\end{cases}
$$

Consider the canonical embedding $\iota: \mathbb{R} \times M_{r, a} \rightarrow \mathbb{R} \times \mathbb{R}^{3}$. Let $\varepsilon$ be a source form on $J^{2}\left(\mathbb{R} \times \mathbb{R}^{3}\right)$, expressed by

$$
\varepsilon=\varepsilon_{x} \omega^{x} \wedge d t+\varepsilon_{y} \omega^{y} \wedge d t+\varepsilon_{z} \omega^{z} \wedge d t
$$

With respect to the associated chart $\left(V_{1}, \Psi_{1}\right), \Psi_{1}=(t, \varphi, \tau)$, on $\mathbb{R} \times M_{r, a}$, we get $J^{2} \iota^{*} \varepsilon=\tilde{\varepsilon}_{\varphi} \eta^{\varphi} \wedge d t+\tilde{\varepsilon}_{\tau} \eta^{\tau} \wedge d t$, where $\eta^{\varphi}=d \varphi-\dot{\varphi} d t, \eta^{\tau}=d \tau-\dot{\tau} d t$, and

$$
\begin{aligned}
\tilde{\varepsilon}_{\varphi} & =\left(\varepsilon_{x} \circ J^{2} \iota\right)\left(-r \sin \varphi+(\tau / 2) \sin (\varphi / 2)-3 \tau \cos ^{2}(\varphi / 2) \sin (\varphi / 2)\right) \\
& +\left(\varepsilon_{y} \circ J^{2} \iota\right)\left(r \cos \varphi+\tau \cos (\varphi / 2)-3 \tau \cos (\varphi / 2) \sin ^{2}(\varphi / 2)\right) \\
& +\left(\varepsilon_{z} \circ J^{2} \iota\right)(\tau / 2) \cos (\varphi / 2), \\
\tilde{\varepsilon}_{\tau} & =\left(\varepsilon_{x} \circ J^{2} \iota\right)\left(\cos (\varphi / 2)-2 \cos (\varphi / 2) \sin ^{2}(\varphi / 2)\right) \\
& +2\left(\varepsilon_{y} \circ J^{2} \iota\right) \sin (\varphi / 2) \cos ^{2}(\varphi / 2)+\left(\varepsilon_{z} \circ J^{2} \iota\right) \sin (\varphi / 2) .
\end{aligned}
$$

Necessary and sufficient conditions (the Helmholtz conditions) for local variationality of $J^{2} \iota^{*} \varepsilon$ now read (cf. Theorem 10),

$$
\begin{aligned}
& \frac{\partial \tilde{\varepsilon}_{\varphi}}{\partial \ddot{\tau}}-\frac{\partial \tilde{\varepsilon}_{\tau}}{\partial \ddot{\varphi}}=0, \quad \frac{\partial \tilde{\varepsilon}_{\varphi}}{\partial \dot{\varphi}}-\frac{d}{d t} \frac{\partial \tilde{\varepsilon}_{\varphi}}{\partial \ddot{\varphi}}=0, \quad \frac{\partial \tilde{\varepsilon}_{\tau}}{\partial \dot{\tau}}-\frac{d}{d t} \frac{\partial \tilde{\varepsilon}_{\tau}}{\partial \ddot{\tau}}=0 \\
& \frac{\partial \tilde{\varepsilon}_{\varphi}}{\partial \dot{\tau}}+\frac{\partial \tilde{\varepsilon}_{\tau}}{\partial \dot{\varphi}}-\frac{d}{d t}\left(\frac{\partial \tilde{\varepsilon}_{\varphi}}{\partial \ddot{\tau}}+\frac{\partial \tilde{\varepsilon}_{\tau}}{\partial \ddot{\varphi}}\right)=0 \\
& \frac{\partial \tilde{\varepsilon}_{\varphi}}{\partial \tau}-\frac{\partial \tilde{\varepsilon}_{\tau}}{\partial \varphi}-\frac{1}{2} \frac{d}{d t}\left(\frac{\partial \tilde{\varepsilon}_{\varphi}}{\partial \dot{\tau}}-\frac{\partial \tilde{\varepsilon}_{\tau}}{\partial \dot{\varphi}}\right)=0 .
\end{aligned}
$$

Moreover, the second de Rham cohomology group of $M_{r, a}$ is trivial, hence $H_{\mathrm{deR}}^{2}(\mathbb{R} \times$ $M_{r, a}$ ) is also trivial. By Corrolary 1 it follows that $J^{2} \iota^{*} \varepsilon$ is automatically globally variational provided (46) are satisfied.

Example 3 (One-dimensional submanifolds of $\mathbb{R}^{m}$ are variational). In Remark 6 we described a class of second-order systems of differential equations which possess any one-dimensional submanifold of $\mathbb{R}^{m}$ as a variational submanifold. Another class of equations of this kind is constructed as follows.

Let $Q$ be an arbitrary embedded one-dimensional submanifold of $\mathbb{R}^{m}$. We search for a source form $\varepsilon$ on $J^{2}\left(\mathbb{R} \times \mathbb{R}^{m}\right)$ such that $J^{2} \iota^{*} \varepsilon$ is (locally) variational. Let $(V, \psi), \psi=\left(u, v^{\alpha}\right), 2 \leq \alpha \leq m$, be a chart on $\mathbb{R}^{m}$ adapted to $Q$, and the canonical embedding $\iota: \mathbb{R} \times Q \rightarrow \mathbb{R} \times \mathbb{R}^{m}$ over $\operatorname{id}_{\mathbb{R}}$, has the equations $t \circ \iota=t, u \circ \iota=\left.u\right|_{V \cap Q}$, $v^{\alpha} \circ \iota=0$. We put $\varepsilon=\varepsilon_{0} \eta \wedge d t+\varepsilon_{\alpha} \omega^{\alpha} \wedge d t$, where $\eta=d u-\dot{u} d t, \omega^{\alpha}=d v^{\alpha}-\dot{v}^{\alpha} d t$, $2 \leq \alpha \leq m$, for arbitrary functions $\varepsilon_{\alpha}$ on $J^{2}\left(\mathbb{R} \times \mathbb{R}^{m}\right)$ and for $\varepsilon_{0}=A+\dot{u}^{2} \ddot{u}$, where $A: V^{1} \rightarrow \mathbb{R}$ is such that $A \circ J^{1} \iota$ depends on coordinate $u$ only. Clearly, $\varepsilon$ may be chosen non-variational. The induced source form has an expression $J^{2} \iota^{*} \varepsilon=$ $\left.\left(A \circ J^{2} \iota+\dot{u}^{2} \ddot{u}\right) \eta\right|_{V \cap Q} \wedge d t$, and one can easily see that $J^{2} \iota^{*} \varepsilon$ is locally variational since the Helmholtz condition (41) is satisfied identically. 
Example 4 (Variational submanifolds: Circles $S_{r}^{1}$ in $\mathbb{R}^{2}$ ). We give a second-order system, which is not variational but every circle $S_{r}^{1}$ in $\mathbb{R}^{2}$ is its variational submanifold. Let $\varepsilon$ be a source form on $J^{2}\left(\mathbb{R} \times \mathbb{R}^{2}\right), \varepsilon=\varepsilon_{\sigma} \omega^{\sigma} \wedge d t$, defined by functions

$$
\varepsilon_{1}=\dot{x}^{2}+\dot{x}^{1}\left(\dot{x}^{1} \ddot{x}^{1}+\dot{x}^{2} \ddot{x}^{2}\right), \quad \varepsilon_{2}=-\dot{x}^{1}+\dot{x}^{2}\left(\dot{x}^{1} \ddot{x}^{1}+\dot{x}^{2} \ddot{x}^{2}\right) .
$$

In the expression (37) we have $A_{1}=\dot{x}^{2}, A_{2}=-\dot{x}^{1}$, and $B_{\sigma \nu}=\dot{x}^{\sigma} \dot{x}^{\nu}, \sigma, \nu=1,2$. It is easy to check that (47) is non-variational since the second condition of (32) is not satisfied. Indeed,

$$
\frac{\partial \varepsilon_{\sigma}}{\partial \dot{x}^{\sigma}}-\frac{d}{d t} \frac{\partial \varepsilon_{\sigma}}{\partial \ddot{x}^{\sigma}}=\sum_{\nu} \dot{x}^{\nu} \ddot{x}^{\nu}+\dot{x}^{\sigma} \ddot{x}^{\sigma}-\frac{d}{d t}\left(\dot{x}^{\sigma}\right)^{2}=\sum_{\nu \neq \sigma} \dot{x}^{\nu} \ddot{x}^{\nu}
$$

remark also that the second condition of (42) is also not satisfied. Consider the polar coordinates $(r, \varphi)$ on $(0,+\infty) \times(0,2 \pi)$, adapted to circles $S_{r}^{1}$ in $\mathbb{R}^{2}$. The mapping $U \ni(t, r, \varphi) \rightarrow\left(t, x^{1}, x^{2}\right) \in V$, given by $t=t, x^{1}=r \cos \varphi, x^{2}=r \sin \varphi$, where

$$
U=\mathbb{R} \times(0,+\infty) \times(0,2 \pi), \quad V=\mathbb{R} \times\left(\mathbb{R}^{2} \backslash\{(x, y) \mid x \geq 0, y=0\}\right),
$$

defines a coordinate transformation between the Cartesian and the polar charts, with respect to which the embedding $\iota: \mathbb{R} \times S_{r}^{1} \rightarrow \mathbb{R} \times \mathbb{R}^{2}$ has an expression $t \circ \iota=t, x^{1} \circ \iota=f^{1}(\varphi)=r \cos \varphi, x^{2} \circ \iota=f^{2}(\varphi)=r \sin \varphi$. One can easily verify that $J^{2} \iota^{*} \varepsilon$ on $J^{2}\left(\mathbb{R} \times S_{r_{0}}^{1}\right)$ is locally variational. Indeed, we get $J^{2} \iota^{*} \varepsilon=\tilde{\varepsilon} \eta \wedge d t$, where $\eta=d \varphi-\dot{\varphi} d t$, and $\tilde{\varepsilon}=r^{4} \dot{\varphi}^{2} \ddot{\varphi}$. The Helmholtz expression for $\tilde{\varepsilon}$ reads,

$$
\frac{\partial \tilde{\varepsilon}}{\partial \dot{\varphi}}-\frac{d}{d t} \frac{\partial \tilde{\varepsilon}}{\partial \ddot{\varphi}}=2 r^{4} \dot{\varphi} \ddot{\varphi}-r^{4} \frac{d}{d t}\left(\dot{\varphi}^{2}\right)=0 .
$$

The system defined by functions (47) belongs to class of mechanical systems with generalised potential force (cf. Dreizler and Lüdde [3]).

Example 5 (Dumped oscillator has no variational submanifold). Consider the equations of motion of the two-dimensional free oscillator in $\mathbb{R} \times \mathbb{R}^{2}$ (cf. Landau and Lifshitz [9]),

$$
m_{11} \ddot{x}+m_{12} \ddot{y}+k_{11} x+k_{12} y=0, \quad m_{12} \ddot{x}+m_{22} \ddot{y}+k_{12} x+k_{22} y=0,
$$

where $k_{11}, k_{12}, k_{22}$ are coefficients of the potential energy, and all the mass coefficients $m_{11}, m_{12}, m_{22}$ are equal 1 . System (48) is variational and coincides with the Euler-Lagrange equations of Lagrange function

$$
\mathscr{L}=\left(m_{11} \dot{x}^{2}+2 m_{12} \dot{x} \dot{y}+m_{22} \dot{y}^{2}-k_{11} x^{2}-2 k_{12} x y-k_{22} y^{2}\right) / 2
$$

(kinetic minus potential energy). Adding to the left-hand sides of (48) the derivatives $\partial F / \partial \dot{x}$ and $\partial F / \partial \dot{y}$ of the dissipative force $F=\left(\alpha_{11} \dot{x}^{2}+2 \alpha_{12} \dot{x} \dot{y}+\alpha_{22} \dot{y}^{2}\right) / 2$, which is a positive definite quadratic form, we get the damped oscillator equations,

$$
\varepsilon_{1}(x, y, \dot{x}, \dot{y}, \ddot{x}, \ddot{y})=0, \quad \varepsilon_{2}(x, y, \dot{x}, \dot{y}, \ddot{x}, \ddot{y})=0,
$$

where $\varepsilon_{1}=\ddot{x}+\ddot{y}+k_{11} x+k_{12} y+\alpha_{11} \dot{x}+\alpha_{12} \dot{y}$, and $\varepsilon_{2}=\ddot{x}+\ddot{y}+k_{12} x+k_{22} y+\alpha_{12} \dot{x}+\alpha_{22} \dot{y}$. System (49) is not variational. Indeed, the Helmholtz conditions (32) for $\varepsilon_{1}, \varepsilon_{2}$ imply that $\alpha_{11}=\alpha_{12}=\alpha_{22}=0$, which contradicts our assumption on dissipative coefficients $\alpha_{i j}$ of the positive definite form $F$.

Consider the canonical embedding $\iota: \mathbb{R} \times Q \rightarrow \mathbb{R} \times \mathbb{R}^{2}$, where $Q$ is a onedimensional submanifold of $\mathbb{R}^{2}$. Denote by $u, v$ the adapted coordinates to $Q$, defined on an open subset of $\mathbb{R}^{2}$, such that $u \circ J^{2} \iota=u, v \circ J^{2} \iota=0$. The pull-back 
$J^{2} \iota^{*} \varepsilon$ of $\varepsilon$, where $\varepsilon=\varepsilon_{1} \omega^{1} \wedge d t+\varepsilon_{2} \omega^{2} \wedge d t, \omega^{1}=d x-\dot{x} d t, \omega^{2}=d y-\dot{y} d t$, has a chart expression $J^{2} \iota^{*} \varepsilon=\tilde{\varepsilon} \eta \wedge d t$, where $\eta=d u-\dot{u} d t$, and

$$
\begin{aligned}
\tilde{\varepsilon} & =\left.\left(\left(\frac{\partial x}{\partial u}\right)^{2}+2 \frac{\partial x}{\partial u} \frac{\partial y}{\partial u}+\left(\frac{\partial y}{\partial u}\right)^{2}\right)\right|_{v=1} \ddot{u} \\
& +\left.\left(\frac{\partial^{2} x}{\partial u^{2}} \frac{\partial x}{\partial u}+\frac{\partial^{2} y}{\partial u^{2}} \frac{\partial x}{\partial u}+\frac{\partial^{2} x}{\partial u^{2}} \frac{\partial y}{\partial u}+\frac{\partial^{2} y}{\partial u^{2}} \frac{\partial y}{\partial u}\right)\right|_{v=1} \dot{u}^{2} \\
& +\left.\left(\alpha_{11}\left(\frac{\partial x}{\partial u}\right)^{2}+2 \alpha_{12} \frac{\partial x}{\partial u} \frac{\partial y}{\partial u}+\alpha_{22}\left(\frac{\partial y}{\partial u}\right)^{2}\right)\right|_{v=1} \dot{u} \\
& +\left.\left(k_{11} x(u, v)+k_{12} y(u, v)\right) \frac{\partial x}{\partial u}\right|_{v=1}+\left.\left(k_{12} x(u, v)+k_{22} y(u, v)\right) \frac{\partial y}{\partial u}\right|_{v=1} .
\end{aligned}
$$

$J^{2} \iota^{*} \varepsilon$ is locally variational if and only if

$$
\begin{aligned}
\frac{\partial \tilde{\varepsilon}}{\partial \dot{u}} & -\frac{d}{d t} \frac{\partial \tilde{\varepsilon}}{\partial \ddot{u}} \\
& =\left.\alpha_{11}\left(\frac{\partial x}{\partial u}\right)^{2}\right|_{v=1}+\left.\left.2 \alpha_{12} \frac{\partial x}{\partial u}\right|_{v=1} \frac{\partial y}{\partial u}\right|_{v=1}+\left.\alpha_{22}\left(\frac{\partial y}{\partial u}\right)^{2}\right|_{v=1}
\end{aligned}
$$

vanishes (cf. (35), Theorem 10). This is, however, not possible as (50) is a positive definite quadratic form which does not vanish for any $Q$. Analogously, we observe that there is no two-dimensional variational submanifold of $\mathbb{R}^{3}$ for the three-dimensional damped oscillator.

Example 6 (Two-dimensional submanifolds of $\mathbb{R}^{m}$ are variational). We generalize Example 3 to two-dimensional submanifolds of $\mathbb{R}^{m}, m \geq 3$. Let $Q$ be an embedded two-dimensional submanifold of $\mathbb{R}^{m}$, and $(V, \psi), \psi=\left(u, v, w^{\alpha}\right), 3 \leq \alpha \leq m$, be a chart on $\mathbb{R}^{m}$ adapted to $Q$. The canonical embedding $\iota: \mathbb{R} \times Q \rightarrow \mathbb{R} \times \mathbb{R}^{m}$ has the equations $t \circ \iota=t, u \circ \iota=\left.u\right|_{V \cap Q}, v \circ \iota=\left.v\right|_{V \cap Q}, w^{\alpha} \circ \iota=0,3 \leq \alpha \leq m$. Let $\varepsilon=\varepsilon_{\sigma} \omega^{\sigma} \wedge d t$ on $J^{2}\left(\mathbb{R} \times \mathbb{R}^{m}\right)$ is defined by

$$
\varepsilon_{\sigma}=B_{\sigma 1} \ddot{u}+B_{\sigma 2} \ddot{v}+B_{\sigma \alpha} \ddot{w}^{\alpha},
$$

where

$$
\begin{array}{lll}
B_{11}=\dot{v}^{2} / 2, & B_{12}=B_{21}=\dot{u} \dot{v}, & B_{1 \alpha}=B_{\alpha 1}=\dot{u} \dot{w}^{\alpha}, \\
B_{22}=\dot{u}^{2} / 2, & B_{2 \alpha}=B_{\alpha 2}=\dot{v} \dot{w}^{\alpha}, & B_{\beta \alpha}=B_{\alpha \beta}=\dot{w}^{\alpha} \dot{w}^{\beta} / 2,
\end{array}
$$

$1 \leq \sigma \leq m, 3 \leq \alpha, \beta \leq m$. It is easy to check that (51) is not variational (cf. Theorem 11). However, the induced source $J^{2} \iota^{*} \varepsilon=\tilde{\varepsilon}_{1} \eta^{1} \wedge d t+\tilde{\varepsilon}_{2} \eta^{2} \wedge d t$, where

$$
\tilde{\varepsilon}_{1}=\frac{1}{2}\left(\dot{v}^{2} \ddot{u}+2 \dot{u} \dot{u} \ddot{v}\right), \quad \tilde{\varepsilon}_{2}=\frac{1}{2}\left(2 \dot{u} \dot{u} \ddot{u}+\dot{u}^{2} \ddot{v}\right),
$$

is locally variational. Moreover, $J^{2} \iota^{*} \varepsilon$ is the Euler-Lagrange form, associated with the Lagrangian

$$
\lambda=\mathscr{L}(\dot{u}, \dot{v}) d t=-\frac{1}{4} \dot{u}^{2} \dot{v}^{2} d t .
$$

Example 7 (A variational submanifold: the sphere $S^{2}$ in $\mathbb{R}^{3}$ ). Let us consider a source form $\varepsilon=\varepsilon_{x} \omega^{x} \wedge d t+\varepsilon_{y} \omega^{y} \wedge d t+\varepsilon_{z} \omega^{z} \wedge d t$ on $J^{2}\left(\mathbb{R} \times \mathbb{R}^{3}\right)$, where $\omega^{x}=d x-\dot{x} d t$, $\omega^{y}=d y-\dot{y} d t, \omega^{z}=d z-\dot{z} d t$, such that

(52) $\varepsilon_{x}=\sqrt{x^{2}+y^{2}+z^{2}}+\ddot{x}, \quad \varepsilon_{y}=\sqrt{x^{2}+y^{2}+z^{2}}+\ddot{y}, \quad \varepsilon_{z}=\sqrt{x^{2}+y^{2}+z^{2}}+\ddot{z}$. 
System (52) is not variational, and we claim that it has a variational submanifold the unit sphere $S^{2}$ in $\mathbb{R}^{3}$. Consider the canonical embedding $\iota: \mathbb{R} \times S^{2} \rightarrow \mathbb{R} \times \mathbb{R}^{3}$, expressed by $t \circ \iota=t, x \circ \iota=\cos \varphi \sin \vartheta, y \circ \iota=\sin \varphi \sin \vartheta, z \circ \iota=\cos \vartheta$, with respect to the canonical coordinates $(t, x, y, z)$ on $\mathbb{R} \times \mathbb{R}^{3}$, and the associated chart $(U, \Phi), \Phi=(t, \varphi, \vartheta)$, on $\mathbb{R} \times S^{2}$. We get $J^{2} \iota^{*} \varepsilon=\tilde{\varepsilon}_{\varphi} \eta^{\varphi} \wedge d t+\tilde{\varepsilon}_{\vartheta} \eta^{\vartheta} \wedge d t$, where $\eta^{\varphi}=d \varphi-\dot{\varphi} d t, \eta^{\vartheta}=d \vartheta-\dot{\vartheta} d t, \tilde{\varepsilon}_{1}=P_{1}+\sin ^{2} \vartheta \ddot{\varphi}, \tilde{\varepsilon}_{2}=P_{2}+\ddot{\vartheta}$, and

$$
\begin{aligned}
& P_{1}=\sin \vartheta(\cos \varphi-\sin \varphi+2 \cos \vartheta \dot{\varphi} \dot{\vartheta}), \\
& P_{2}=\sin \varphi \cos \vartheta+\cos \varphi \cos \vartheta-\sin \vartheta-\sin \vartheta \cos \vartheta \dot{\varphi}^{2} .
\end{aligned}
$$

A direct calculation shows that $J^{2} \iota^{*} \varepsilon$ is locally variational. Moreover, $J^{2} \iota^{*} \varepsilon$ is the Euler-Lagrange form, associated with the Lagrangian $\tilde{\mathscr{L}} d t$, where

$$
\tilde{\mathscr{L}}=-\frac{1}{2}\left(\sin ^{2} \vartheta \dot{\varphi}^{2}+\dot{\vartheta}^{2}\right)+\cos \varphi \sin \vartheta+\sin \varphi \sin \vartheta+\cos \vartheta .
$$

Note that the Lagrange function $\tilde{\mathscr{L}}$ comes from the mechanical Lagrangian $\mathscr{L} d t$, defined on $J^{1}\left(\mathbb{R} \times \mathbb{R}^{3}\right)$, where $\mathscr{L}=-\left(\dot{x}^{2}+\dot{y}^{2}+\dot{z}^{2}\right) / 2+x+y+z$ satisfies $\tilde{\mathscr{L}}=\mathscr{L} \circ J^{1} \iota$.

Example 8 (Gyroscopic type system). The equations of motion of the 3-dimensional gyroscopic type system in the Euclidean space $\mathbb{R} \times \mathbb{R}^{3}$ read (cf. Landau and Lifshitz [9]),

$$
\ddot{x}=\alpha \dot{y}+\beta \dot{z}, \quad \ddot{y}=-\alpha \dot{x}+\gamma \dot{z}, \quad \ddot{z}=-\beta \dot{x}-\gamma \dot{y},
$$

where $\alpha, \beta$, and $\gamma$ are arbitrary functions of the positions $x, y, z$. Note the righthand side of (53) belongs to a class of mechanical forces, expressible as a vector product of velocity and position vectors; basic examples are the Lorentz force, or the Coriolis force (cf. Dreizler and Lüdde [3]). Computing the Helmholtz expressions (32), we observe that the system, $\varepsilon_{1}=\ddot{x}-\alpha \dot{y}-\beta \dot{z}, \varepsilon_{2}=\ddot{y}+\alpha \dot{x}-\gamma \dot{z}, \varepsilon_{3}=\ddot{z}+\beta \dot{x}+\gamma \dot{y}$, on $J^{2}\left(\mathbb{R} \times \mathbb{R}^{3}\right)$ is locally variational if and only if

$$
\frac{\partial \alpha}{\partial z}-\frac{\partial \beta}{\partial y}+\frac{\partial \gamma}{\partial x}=0
$$

Let $Q$ be an embedded two-dimensional submanifold of $\mathbb{R}^{3}, \iota: \mathbb{R} \times Q \rightarrow \mathbb{R} \times \mathbb{R}^{3}$ the canonical embedding. Denote by $u, v$, and $w$ the adapted coordinates to $Q$, defined on an open subset of $\mathbb{R}^{3}$, such that $\iota$ has the equations $u \circ \iota=\left.u\right|_{Q}, v \circ \iota=\left.v\right|_{Q}$, and $w \circ \iota=0$. Then $J^{2} \iota^{*} \varepsilon=\tilde{\varepsilon}_{1} \eta^{1} \wedge d t+\tilde{\varepsilon}_{2} \eta^{2} \wedge d t$, where $\eta^{1}=d u-\dot{u} d t, \eta^{2}=d v-\dot{v} d t$, and

where

$$
\tilde{\varepsilon}_{1}=P_{1}+Q_{11} \ddot{u}+Q_{12} \ddot{v}, \quad \tilde{\varepsilon}_{2}=P_{2}+Q_{12} \ddot{u}+Q_{22} \ddot{v},
$$

$$
\begin{aligned}
& P_{1}=\left(\frac{\partial f^{x}}{\partial u}\right)\left(\frac{\partial^{2} f^{x}}{\partial u^{2}}\right) \dot{u}^{2}+2\left(\frac{\partial f^{x}}{\partial u}\right)\left(\frac{\partial^{2} f^{x}}{\partial v \partial u}\right) \dot{u} \dot{v}+\left(\frac{\partial f^{x}}{\partial u}\right)\left(\frac{\partial^{2} f^{x}}{\partial v^{2}}\right) \dot{v}^{2} \\
& +\left(\frac{\partial f^{y}}{\partial u}\right)\left(\frac{\partial^{2} f^{y}}{\partial u^{2}}\right) \dot{u}^{2}+2\left(\frac{\partial f^{y}}{\partial u}\right)\left(\frac{\partial^{2} f^{y}}{\partial v \partial u}\right) \dot{u} \dot{v}+\left(\frac{\partial f^{y}}{\partial u}\right)\left(\frac{\partial^{2} f^{y}}{\partial v^{2}}\right) \dot{v}^{2} \\
& +\left(\frac{\partial f^{z}}{\partial u}\right)\left(\frac{\partial^{2} f^{z}}{\partial u^{2}}\right) \dot{u}^{2}+2\left(\frac{\partial f^{z}}{\partial u}\right)\left(\frac{\partial^{2} f^{z}}{\partial v \partial u}\right) \dot{u} \dot{v}+\left(\frac{\partial f^{z}}{\partial u}\right)\left(\frac{\partial^{2} f^{z}}{\partial v^{2}}\right) \dot{v}^{2} \\
& +\left(\frac{\partial f^{y}}{\partial u} \frac{\partial f^{x}}{\partial v}-\frac{\partial f^{x}}{\partial u} \frac{\partial f^{y}}{\partial v}\right) \alpha \dot{v}+\left(\frac{\partial f^{z}}{\partial u} \frac{\partial f^{x}}{\partial v}-\frac{\partial f^{x}}{\partial u} \frac{\partial f^{z}}{\partial v}\right) \beta \dot{v} \\
& +\left(\frac{\partial f^{z}}{\partial u} \frac{\partial f^{y}}{\partial v}-\frac{\partial f^{y}}{\partial u} \frac{\partial f^{z}}{\partial v}\right) \gamma \dot{v},
\end{aligned}
$$




$$
\begin{aligned}
& P_{2}=\left(\frac{\partial f^{x}}{\partial v}\right)\left(\frac{\partial^{2} f^{x}}{\partial u^{2}}\right) \dot{u}^{2}+2\left(\frac{\partial f^{x}}{\partial v}\right)\left(\frac{\partial^{2} f^{x}}{\partial v \partial u}\right) \dot{u} \dot{v}+\left(\frac{\partial f^{x}}{\partial v}\right)\left(\frac{\partial^{2} f^{x}}{\partial v^{2}}\right) \dot{v}^{2} \\
& +\left(\frac{\partial f^{y}}{\partial v}\right)\left(\frac{\partial^{2} f^{y}}{\partial u^{2}}\right) \dot{u}^{2}+2\left(\frac{\partial f^{y}}{\partial v}\right)\left(\frac{\partial^{2} f^{y}}{\partial v \partial u}\right) \dot{u} \dot{v}+\left(\frac{\partial f^{y}}{\partial v}\right)\left(\frac{\partial^{2} f^{y}}{\partial v^{2}}\right) \dot{v}^{2} \\
& +\left(\frac{\partial f^{z}}{\partial v}\right)\left(\frac{\partial^{2} f^{z}}{\partial u^{2}}\right) \dot{u}^{2}+2\left(\frac{\partial f^{z}}{\partial v}\right)\left(\frac{\partial^{2} f^{z}}{\partial v \partial u}\right) \dot{u} \dot{v}+\left(\frac{\partial f^{z}}{\partial v}\right)\left(\frac{\partial^{2} f^{z}}{\partial v^{2}}\right) \dot{v}^{2} \\
& +\left(\frac{\partial f^{y}}{\partial u} \frac{\partial f^{x}}{\partial v}-\frac{\partial f^{x}}{\partial u} \frac{\partial f^{y}}{\partial v}\right) \alpha \dot{v}+\left(\frac{\partial f^{z}}{\partial u} \frac{\partial f^{x}}{\partial v}-\frac{\partial f^{x}}{\partial u} \frac{\partial f^{z}}{\partial v}\right) \beta \dot{v} \\
& +\left(\frac{\partial f^{z}}{\partial u} \frac{\partial f^{y}}{\partial v}-\frac{\partial f^{y}}{\partial u} \frac{\partial f^{z}}{\partial v}\right) \gamma \dot{v}
\end{aligned}
$$

and

$$
\begin{aligned}
Q_{11} & =\left(\frac{\partial f^{x}}{\partial u}\right)^{2}+\left(\frac{\partial f^{y}}{\partial u}\right)^{2}+\left(\frac{\partial f^{z}}{\partial u}\right)^{2}, \quad Q_{22}=\left(\frac{\partial f^{x}}{\partial v}\right)^{2}+\left(\frac{\partial f^{y}}{\partial v}\right)^{2}+\left(\frac{\partial f^{z}}{\partial v}\right)^{2}, \\
Q_{12} & =\frac{\partial f^{x}}{\partial u} \frac{\partial f^{x}}{\partial v}+\frac{\partial f^{y}}{\partial u} \frac{\partial f^{y}}{\partial v}+\frac{\partial f^{z}}{\partial u} \frac{\partial f^{z}}{\partial v} .
\end{aligned}
$$

The source form $J^{2} \iota^{*} \varepsilon$ satisfies the Helmholtz conditions (Theorem 11) for arbitrary functions $\alpha, \beta, \gamma$ depending on the positions $x, y, z$. Hence any two-dimensional submanifold of $\mathbb{R}^{3}$ is a variational submanifold for the gyroscopic type system (53).

\section{REFERENCES}

[1] I.M. Anderson and T. Duchamp, On the existence of global variational principles, Am. J. Math. 102 (1980), 781-867.

[2] J. Brajerčík and D. Krupka, Variational principles for locally variational forms, J. Math. Phys. 46 (2005), 052903.

[3] R.M. Dreizler and C.S. Lüdde, Theoretical Mechanics, Theoretical Physics Vol. 1, Graduate Texts in Physics, Springer-Verlag, Berlin, 2011.

[4] D. Krupka, On the local structure of the Euler-Lagrange mapping of the calculus of variations, in: Proc. Conf. Diff. Geom. Appl., Charles University, Prague, pp. 181-188, 1981; arXiv:math-ph/0203034

[5] D. Krupka, Variational sequences on finite order jet spaces, in: Diff. Geom. Appl., Prof. Conf., 27 August - 2 September, 1989, Czechoslovakia, World Scientific, Singapore, pp. 236-254, 1990.

[6] D. Krupka, Variational sequences in mechanics, Calc. Var. 5 (1997), 557-583.

[7] D. Krupka, Introduction to Global Variational Geometry, Atlantis Press, Amsterdam, 2015.

[8] D. Krupka and D. Saunders (Eds.), Handbook of Global Analysis, Elsevier, Amsterdam, 2008.

[9] L.D. Landau and E.M. Lifshitz, Mechanics, 2nd Edition, Course of Theoretical Physics, Vol. 1, Pergamon Press, Oxford, 1969.

[10] W. Sarlet, The Helmholtz conditions revisited. A new approach to the inverse problem of Lagrangian dynamics, J. Phys. A: Math. Gen. 15 (1982), 1503-1517.

[11] F. Takens, A global version of the inverse problem of the calculus of variations, J. Diff. Geom. 14 (1979), 543-562.

[12] Z. Urban and D. Krupka, Variational sequences in mechanics on Grassmann fibrations, Acta Appl. Math. 112, No. 2 (2010), 225-249.

[13] Z. Urban, Variational Principles for Immersed Submanifolds, in: D. Zenkov (Ed.), The Inverse Problem of the Calculus of Variations, Atlantis Press, Amsterdam, pp. 103-170, 2015.

[14] N. Voicu and D. Krupka, Canonical variational completion of differential equations, J. Math. Phys. 56 (2015), 043507.

[15] J. Volná and Z. Urban, The interior Euler-Lagrange operator in field theory, Math. Slovaca 65, No. 6 (2015), 1427-1444.

[16] F.W. Warner, Foundations of Differentiable Manifolds and Lie Groups, 2nd Ed., Springer, New York, 1983. 
Demeter Krupka

Lepage Research Institute, University of Presov

17th November 1, Presov, Slovakia

E-mail address: demeter.krupka@lepageri.eu

ZBYNĔK URBan and Jana Volná

Department of Mathematics and Descriptive Geometry

VŠB-Technical University of Ostrava

17. listopadu 15, 70833 Ostrava-Poruba, Czech Republic

E-mail address: zbynek.urban@vsb.cz; jana.volna@vsb.cz 